\title{
Jeux dialogiques et processus discursif. Conséquences du débat entre Habermas et Brandom
}

\author{
YAËL SEBBAN Collège de l'Outaouais
}

RÉSUMÉ : Dans cet article, nous soutenons que Habermas réduit, dans l'essai critique sur Brandom inclus dans Vérité et justification (2001), la communication à sa forme étroitement dialogique et, ce faisant, tend à ignorer que le processus discursif est avant tout, pour Brandom, le produit d'une coordination partagée de différentes perspectives individuelles contribuant à faire avancer ce jeu de production et de demande de raisons. Nous proposons, d'une part, de procéder à une analyse critique de la position de Habermas, et d'autre part, de rendre justice à Brandom en soulignant la spécificité du processus communicatif discursif.

ABSTRACT: In this article, I maintain that Habermas, in his critical essay on Brandom included in Truth and Justification (2003), reduces communication to its narrowly dialogical form, and in doing so tends to ignore the fact that the discursive process is, above all, for Brandom, the product of a shared coordination of different individual perspectives contributing to advancing the game of giving and asking one another for reasons. On the one hand, I propose to carry out a critical analysis of Habermas' position and, on the other hand, to do justice to Brandom by emphasizing the specificity of the discursive communicative process.

Mots clés: Brandom, Habermas, communication, logique dialogique, inférentialisme, processus discursif, perspectivisme, objectivité

Dialogue 59 (2020), 305-344

(C) The Author(s), 2020. Published by Cambridge University Press on behalf of the Canadian Philosophical Association/I'Association canadienne de philosophie doi:10.1017/S0012217320000207 


\section{Introduction}

Dans Philosophy and Phenomenological Research (1997, vol. 57, $\mathrm{n}^{\mathrm{O}}$ 1), une discussion s'articule autour de Rendre explicite, l'ouvrage majeur de Robert Brandom ${ }^{1}$. Richard Rorty (1997) ouvre le débat entre Jürgen Habermas et Robert Brandom sur la question de l'objectivité de nos prétentions à la validité afin de clarifier le rôle des pratiques sociales au cœur de nos échanges discursifs. Le débat entre les deux philosophes se poursuit en l'an 2000 dans le European Journal of Philosophy (voir Brandom, 2000a et Habermas, 2000). Habermas reprendra sa contribution à ce débat dans un chapitre de Vérité et justification où il présente une analyse détaillée et critique de la pragmatique normative de Brandom ${ }^{2}$. La critique de Habermas est dirigée à l'encontre de la compréhension prétendument objectiviste de la pratique discursive proposée par Brandom. Habermas amorce cette critique du point de vue intersubjectiviste et pense trouver dans la relation «je-tu», distincte de la relation «je-il», les conditions spécifiques d'une compréhension mutuelle atteinte au moyen du langage. Habermas reproche ainsi au modèle de communication de Brandom de ne pas être «dialogique» au sens où il requerrait un «face à face» entre un locuteur et un interlocuteur, et de réduire le rôle de la seconde personne à celui d'un observateur, ce qui correspond au point de vue d'une troisième personne. Nous proposons ici, d'une part, de mener une analyse critique de la position de Habermas telle qu'elle est exposée dans l'essentiel de son essai — et plus particulièrement dans la section V(2) (Habermas, 2001, p. 112-117), axée sur la structure dialogique de la communication. D'autre part, nous voulons rendre justice à Brandom en soulignant la spécificité du processus communicatif discursif conçu comme un jeu d'offre et de demande de raisons, au sein duquel «Dire "nous" dans ce sens, c'est nous situer, nous-mêmes et mutuellement dans l'espace des raisons, en donnant et en exigeant des raisons de nos attitudes et de nos actes» (Branbom, 2010, p. 59). Dans ce qui suit, nous laisserons en grande partie ouverte la question de savoir si Brandom adopte réellement une approche objectiviste ${ }^{3}$ de la pratique discursive. Nous questionnerons la conception de l'intersubjectivité défendue par Habermas, qui privilégie tantôt sa dimension strictement dialogique, où la relation entre l'acte de parole de l'ego et la prise de position de l'alter ego forme la plus petite unité analytique dans des contextes d'interaction, tantôt sa dimension discursive et critique, où la

1 Robert Brandom, Rendre explicite : raisonnement, représentation et engagement discursif, vol. I, Paris, Cerf (2010 [1994]). Le volume II étant paru, pour la traduction française, en 2011, nous citons en employant les références Brandom, 2010 et Brandom, 2011.

Jürgen Habermas, «De Kant à Hegel : la pragmatique linguistique de Robert Brandom», dans Vérité et justification, chapitre 3 (2001, p. 81-124). Ce chapitre est une traduction de l'article de 2000.

3 Voir la critique de Rorty (1997, p. 173-175). 
distribution symétrique des opportunités en termes d'obligations de disposer de justifications et de droits de les contester permet à tout participant à la discussion de faire valoir son point de vue et de remettre en question les opinions d'autrui, ainsi que les implications théoriques qui découlent de cette conception de l'intersubjectivité. Une bonne compréhension de l'intersubjectivité, en particulier du processus de communication par lequel elle s'établit, est en jeu. Nous soutenons que Habermas semble perdre de vue la pratique discursive en tant que telle en exigeant une forme plus robuste d'interaction ${ }^{4}$, celle-ci impliquant la coordination interactive ou dialogique des intentions et des actions des seuls agents directement impliqués dans la conversation. En réduisant la pratique discursive aux échanges entre le locuteur et son interlocuteur, on risque d'ignorer que le processus discursif est avant tout le produit d'une coordination partagée des évaluations des perspectives différentes des interlocuteurs, celles-ci ne pouvant être rendues intelligibles que dans le contexte d'une interprétation mutuelle ou d'une relation sur le mode interpersonnel du «je-tu», comme le préconise Brandom. En effet, la pratique discursive qui consiste à donner et à demander des raisons, à partir de laquelle sont extraites les relations inférentielles entre contenus propositionnels qui jouent le rôle de prémisses et de conclusions de nos raisonnements, doit être comprise, selon Brandom, «en référence à la dimension sociale de la communication des raisons et de l'évaluation de la portée des raisons données par autrui» (2011, p. 883).

Nous explicitons, dans la première partie de cet article (section 2), le rapport entre sémantique et pragmatique, la pratique discursive procédant «à une mise en route du réseau des relations inférentielles disposées dans la langue» ${ }^{5}$, celles-ci déterminant le contenu des assertions sur la base de ce jeu continu de marquage au score, afin de montrer que Brandom évoque bien le processus conversationnel sur le mode du «je-tu», mais sans lier cette pratique à la sémantique de la même manière que le requiert la logique dialogique. Il sera donc question de voir si cette présumée lacune soulevée par Habermas, c'est-à-dire l'absence d'une dimension strictement dialogique, entrave chez Brandom le processus discursif de la communication. Nous situons, dans la section 3 de cet article, la critique que Habermas adresse à Brandom et dans laquelle il propose de corriger la tendance objectiviste de ce dernier. Nous procédons enfin, dans les sections 4 et 5 , à une analyse critique de la position de Habermas et proposons, comme solution, d'envisager avec Brandom le processus discursif dans sa dimension séquentielle et perspectiviste.

4 Piet Strydom (2006) soutient ce point, mais il s'en tient à une analyse critique de la position de Habermas, alors que nous visons ici à répondre à cette critique en explicitant la spécificité du processus discursif théorisé par Brandom.

5 Habermas (2001, p. 87). 


\section{Le modèle de communication de Brandom et le problème dialogique}

Brandom (2010, p. 288) estime que la sémantique doit répondre à la pragmatique. Dans la continuité de Wittgenstein $(2005, \S 43, \S 138)$, selon lequel la signification d'une expression relève de son usage, nous ne pouvons pas séparer la sémantique de la pragmatique. L'un des engagements méthodologiques qui oriente ce projet est d'expliquer les significations des expressions linguistiques en termes de conditions d'usage correct des énoncés (Brandom, 2010, p. 33). Dès lors, ce qu'un sémanticien associe à des états intentionnels ou à des expressions ne peut être qualifié de contenu sémantique que dans la mesure où il joue un rôle dans la pratique qui gouverne ces états et expressions (ibid., p. 194). Il est certes possible d'associer toutes sortes d'objets abstraits à des langages formels, par exemple dans la théorie des modèles. Cependant, une telle association n'est proprement sémantique qu'à la condition de déterminer comment ces symboles sont utilisés correctement. Par exemple, une interprétation du calcul des prédicats ne peut être qualifiée de sémantique que dans la mesure où elle permet de donner une représentation de la notion d'inférence valide, c'est-à-dire «une manière de dire ce qui suit de quoi» ou une représentation de ce qu'il est correct de faire (ibid., p. 194). Notons cependant que la philosophie du langage de Brandom est d'abord sémantique et que son échelle d'analyse est propositionnelle. S'il s'agit de fonder la sémantique dans une pragmatique, de montrer que «la pragmatique et la sémantique entretiennent des relations intimes» (ibid., p. 43), c'est toujours en supposant que le langage est une pratique à justifier : le contenu de sens de nos expressions est entièrement dépendant de ce que nous pouvons faire avec elles. Une théorie sémantique n'est alors pertinente que si elle permet de rendre compte de ce que nous faisons ou devons faire lorsque nous parlons ou pensons ${ }^{6}$. L'enjeu est alors de «rendre explicite ce que nous sommes» (Brandom, 2010, p. 59) et la manière dont nous nous comprenons. Ceci s'explique parce que nos «transactions» (ibid., p. 59) signifient quelque chose pour nous et ont un contenu conceptuel qui fait en sorte que nous les comprenons. C'est donc bien en termes de «contenu», plus précisément de contenu conceptuel et de contenu propositionnel dans le cas des pratiques discursives, que doivent s'analyser nos états mentaux dits «intentionnels», tels que croire, penser, etc. (Brandom, 2010, p. 207). Les états de croyance et les actes de jugement peuvent ainsi être exprimés par des assertions. Les expressions linguistiques qui ont la signification par défaut d'une assertion sont des phrases (déclaratives). Ce qu'il s'agit de rendre explicite, c'est donc le contenu conceptuel de nos affirmations et de nos états intentionnels, ce qui peut tenir lieu de raison de nos pratiques dans la perspective d'une dimension sociale ou le fait

6 Voir John McDowell (1997, p. 157) : «Semantic terms are not intelligible apart from how they pull their weight in enabling us to make sense of what speakers $d o$, on the way we make sense of rational behaviour in general». 
de «donner et de demander des raisons sur le mode interpersonnel» (Brandom, 2011, p. 883). En effet, Brandom souligne que

Être une raison signifie être compris, en premier lieu, dans les termes de ce que c'est pour une communauté, que traiter quelque chose en pratique comme une telle raison, sur le plan pratique des raisons pour l'action aussi bien que sur le plan doxastique des raisons pour nos affirmations (ibid., p. 475).

Brandom retient ainsi de son maître Wilfrid Sellars (1956) que nous nous inscrivons, en tant que sujets rationnels, dans «l'espace des raisons» et que les comportements des agents sont des comportements rationnels toujours susceptibles de justification. Le concept de Sellars est un concept kantien. Cet espace, où nous nous situons, tient lieu de «tribunal» ${ }^{7}$ devant lequel nous pouvons justifier nos assertions lorsque nous énonçons telle ou telle inférence. Brandom pose clairement ce réquisit de justification à nos pratiques. Pratiquer, c'est alors s'engager dans l'espace des raisons de manière justifiée, c'est-à-dire en étant habilité à le faire : «Les pratiques qui confèrent un contenu propositionnel — et d'autres types de contenu conceptuel - contiennent implicitement des normes relatives à la manière dont il est correct d'utiliser des expressions, dans quelles circonstances il est approprié d'accomplir différents actes de langage, et quelles sont les conséquences appropriées de tels actes» (Brandom, 2010, p. 35). Ainsi qu'il le précise ailleurs, ce sont alors les pratiques «d'offre et de demande de raisons» qui «confèrent un contenu conceptuel aux performances, expressions et états qui s'inscrivent comme il convient dans ces pratiques» (Brandom, 2009, p. 18). Aussi, c'est dans l'espace des raisons que l'on peut expliciter le contenu conceptuel de nos pratiques, et ce qui confère un sens aux contenus conceptuels est bien leur articulation inférentielle. La sémantique de Brandom donne une priorité logique aux propositions parce que celles-ci sont les éléments privilégiés des inférences ${ }^{8}$. En ce sens, le contenu conceptuel est à comprendre à partir de son rôle dans le raisonnement : ce contenu conceptuel est justifiable s'il joue un rôle de prémisse ou de conclusion dans les inférences de nos raisonnements.

7 Il s'agit du concept introduit dans la préface de la première édition de la Critique de la raison pure, où Kant énonce «l'invitation faite à la raison d'entreprendre à nouveau la plus difficile de toutes ses tâches, celle de la connaissance de soi-même, et d'instituer un tribunal qui la garantisse dans ses prétentions légitimes et puisse en retour condamner toutes ses prétentions, non pas d'une manière arbitraire, mais au nom de ses lois éternelles et immuables. Or, ce tribunal n'est autre chose que la Critique de la Raison pure elle-même» (1944, p. 7, nous soulignons).

8 Voir John MacFarlane (2010, p. 85) : «Brandom repeatedly emphasizes that the inferentialist's fundamental semantic concepts can be explicated in terms of properties for performing a certain kind of action - the action of inferring, of drawing a conclusion from premises». 
La tâche de la sémantique qu'il nomme «sémantique inférentielle» consiste alors à élucider le rôle inférentiel de tout contenu conceptuel en tant que prémisse ou conclusion de nos raisonnements :

Être explicite, au sens conceptuel, c'est jouer un rôle spécifiquement inférentiel. Dans le cas le plus fondamental, c'est être doté d'un contenu propositionnel au sens où l'on est prêt à servir à la fois de prémisse et de conclusion dans des inférences (Brandom, 2009, p. 24).

Brandom (2009, p. 69) articule initialement la notion de rôle inférentiel d'une expression en reprenant cette idée de Michael Dummett (1981) selon laquelle la signification d'un terme est donné par les circonstances dans lesquelles il est correctement appliqué, énoncé ou utilisé et par les conséquences appropriées de son application, de son énonciation ou de son usage (Dummett, 1981). Il explique ensuite ces concepts à partir des deux statuts déontiques ou normatifs à l'origine desquels les formes d'inférence sont dérivées, à savoir : les engagements et les permissions ou habilitations ${ }^{9}$ à ces engagements. Ces statuts déontiques sont, par la suite, pensés dans le cadre social de leur interaction à partir des deux attitudes normatives - telles qu'attribuer ou imputer un engagement ou une permission à autrui et reconnaître soi-même un engagement ou une permission — qui constituent les deux mouvements de base de la procédure de marquage au score déontique ${ }^{10}$ (Brandom, 2010, p. 328-329). Brandom souligne ici la dimension normative ${ }^{11}$ de la pragmatique, celle-ci relevant de ce qu'il est correct ou incorrect de juger et d'inférer dans un cadre interlocutif. En effet, si Brandom endosse non seulement la thèse wittgensteinienne selon laquelle les significations des expressions linguistiques doivent être expliquées en termes d'usage, il donne également un prolongement à une autre idée phare de ce philosophe qui soutenait que

les états et les actes à contenu intentionnel ont une portée pragmatique essentiellement normative [...] [il faut] les comprendre comme des états et des actes qui nous engagent ou nous obligent à agir et à penser de diverses manières (Brandom, 2010, p. 73).

Aussi, comme le rappelle Brandom, «le monde naturel ne surgit pas avec, en lui, des engagements et des habilitations; ce sont là des produits de l'activité

\footnotetext{
9 Nous traduisons «entitlement» par permission, autorisation ou habilitation.

10 Nous emploierons indifféremment les expressions de marquage ou de pointage au score.

11 Voir Brandom (1997, p. 193) : «The concept of meaning is a normative concept. The theoretical explanatory job distinctive of concepts of meanings or conceptual content is to settle how it would be correct to use words or to apply concepts, how those meanings determine how they ought to be used, how those who use concepts with those concepts are committed thereby to apply them».
} 
humaine» (ibid., p. 35) qui résultent de notre capacité à accomplir différents types d'actes de langage, savoir-faire pratiques qui mettent en jeu implicitement des normes. Par conséquent, toute pratique sociale doit, pour être considérée comme discursive, inclure dans son jeu d'offre et de demande de raisons l'attribution par un interprète et la reconnaissance ou la contraction par l'interprété des deux statuts normatifs qui les unissent : les engagements et les habilitations aux engagements (Brandom, 2009, p. 201). Le contexte de l'échange discursif consiste donc dans «l'évaluation de la manière dont les jugements d'un individu peuvent servir de raisons pour un autre individu» (Brandom, 2011, p. 883). Brandom clarifie ici la dimension sociale de la communication des raisons et de l'évaluation de la portée des raisons par autrui :

On soutiendra que la capacité de la pratique à conférer un tel contenu tient essentiellement au fait qu'il ne s'agit pas seulement d'une pratique sociale, mais bien d'une pratique sociale linguistique, en ce que son cœur est constitué par la communication, et plus spécifiquement par l'interprétation que les praticiens fournissent de leurs assertions respectives (2010, p. 312).

La normativité des engagements et des habilitations est ainsi instituée par les agents impliqués dans ce processus qui agissent à titre de marqueurs (scorekeepers) ${ }^{12}$ qui comptabilisent les points et qui suivent le cours de ce qui est dit par chaque participant au jeu en leur attribuant des engagements et des habilitations. Brandom introduit alors une distinction entre une justification en première et en troisième personne. Nous devons nous reconnaître nous-mêmes comme habilité à faire telle ou telle chose, mais il faut aussi que la société via l'interprète ou le marqueur nous attribue cette habilitation. Or, «de ces deux attitudes, c'est l'attribution qui est l'attitude fondamentale» (Brandom, 2010, p. 329). Or, le problème avec cette insistance sur ce que Brandom nomme la «théorie de l'évaluation communautaire» (ibid., p. 111) est, comme le lui reprochera Habermas (2001), qu'elle introduit un processus d'évaluation externe qui prévaut sur la dynamique interne du dialogue. Brandom affirme ainsi que «comprendre un acte de langage - en saisir la signification discursive - c'est être capable d'attribuer les justes engagements en réponse. C'est savoir en quoi il modifie le score des engagements et autorisations qui incombent à qui en est l'auteur et à ceux qui en sont les destinataires» (2009, p. 174). Aussi Brandom consacre-t-il une attention particulière aux «sanctions normatives» qui évaluent les «attitudes normatives» afin de déterminer ce que signifie «agir droitement» et punir «ceux qui agissent comme ils ne sont pas (censés être) habilités à agir, et ceux qui n'agissent pas comme ils sont (censés être) engagés à le faire» (Brandom, 2011, p. 328-329). Par conséquent, un locuteur qui

12 Chaque joueur qui comptabilise les points dans un match joue aussi le rôle d'un arbitre qui marque les points effectués par chacun des interlocuteurs. Nous traduirons par marqueur. 


\section{Dialogue}

se dit engagé à faire telle ou telle action autorise le marqueur à lui imputer cet engagement. C'est finalement cette pratique qui consiste à reconnaître et à contracter des engagements et des habilitations en se les faisant attribuer par un autre joueur marquant les points et, lui-même, reconnaissant et contractant à son tour des engagements et habilitations identiques ou différents, qui constitue le pointage ou le marquage déontique. Le marquage déontique se présente donc comme essentiellement social. Dans une conversation, la comptabilité des points correspond aux engagements et habilitations associés à chaque participant. Chaque participant à la conversation marque les points de chacun des participants (incluant lui-même) et les intervenants d'une pratique discursive suivent les coups des engagements et autorisations de chacun des autres joueurs. Les personnes prenant part à une conversation tiennent donc les comptes de leurs propres engagements et habilitations ainsi que ceux des autres. Chaque fois que l'un des participants adopte implicitement, reconnaît explicitement ou attribue un engagement ou une habilitation, cela change le pointage. Les actes de langage ont pour effet de modifier le score déontique en induisant un changement quant aux engagements et habilitations qu'il convient d'attribuer, non seulement à l'auteur de l'acte de langage, mais encore à ceux à qui il est adressé. Brandom fonde ainsi sa théorie pragmatique sur l'idée que l'emploi d'une expression linguistique représente la manière de changer le score d'une conversation. En mettant l'accent sur la dimension normative du contenu, Brandom définit la portée pragmatique d'un acte de langage comme la manière dont celui-ci affecte l'évolution du pointage de la conversation au sein de laquelle il s'inscrit (Brandom, 2010, p. 287). C'est donc la dimension inférentielle de ces contraintes pragmatiques qui définit le contenu de ce qui est dit : par exemple, chaque engagement attribué ou reconnu s'accompagne de la reconnaissance implicite d'autres engagements présupposés ou impliqués. Par conséquent, pour déterminer précisément quels sont les engagements et les permissions ou habilitations qui devraient être attribués, reconnus et contractés par les participants à la conversation, il faut mettre en œuvre ces relations sémantiques entre engagements et permissions, qui sont d'abord des normes pragmatiques, en les explicitant en termes de relations inférentielles (Brandom, 2010, p. 333-334; 2009, p. 205) entre les contenus assertés, comme suit :

1) Des relations inférentielles au sein desquelles les engagements sont préservés. Il est question ici des inférences déductives où tout locuteur engagé envers $p$ est engagé envers $q$. Par conséquent, si nous reconnaissons explicitement que $q$ est une déduction de $p$ et que nous sommes aussi engagés à $p$, alors nous devons aussi reconnaître un engagement à $q$. Quand nous assertons «cette tomate est rouge», nous nous engageons aussi à l'égard de la vérité du contenu «cette tomate n'est pas verte». Nous nous sommes engagés vis-à-vis d'un contenu parce que nous sommes engagés envers un autre contenu. Nous sommes également engagés envers ce qui se situe - toujours par relation déductive - en amont de notre assertion, par exemple le contenu «il y a une tomate». 
2) Des relations inférentielles permissives au sein desquelles des permissions ou des autorisations sont préservées. Il est question ici d'inférences inductives où être engagé envers $p$ peut être une raison pour s'engager envers $q$; il est permis d'asserter $q$ à partir de $p$ si nous pouvons inférer $q$. Quand nous assertons «ceci est une tomate mûre», nous pouvons nous baser sur ce contenu pour arriver à d'autres contenus, par le biais de conditions d'arrièreplan présupposées, comme «si elle est exposée au soleil, elle rougira» (Brandom, 1983, p. 641).

3) L'interaction entre 1 et 2 engendre des relations matérielles de négation ou d'incompatibilité entre contenus (Brandom, 2010, p. 319 et p. 334). Il est question ici de l'inférence modale où un engagement envers $p$ interdit une permission pour un engagement envers $q$; $p$ est incompatible avec $q$ si un engagement envers $p$ interdit ou exclut un engagement envers $q$ (Brandom, 2009, p. 204). L'engagement envers $p$ exclut l'engagement envers $\neg p$ ou tout contenu conceptuel duquel on pourrait inférer $\neg p$.

Les relations inférentielles constitutives des contenus assertés n'existent qu'en rapport avec les normes pragmatiques elles-mêmes attribuées et instituées par d'autres attitudes normatives. Les propriétés inférentielles qui définissent la nature sémantique d'un contenu sont donc fondamentalement des propriétés pragmatiques socialement instituées ${ }^{13}$ dans et par les pratiques linguistiques et discursives de marquage au score (Brandom, 1983, p. 646-647). Ces propriétés expriment ce que nous devons faire et ce que nous pouvons reconnaître ou faire inférentiellement en ayant énoncé ce contenu; ces propriétés n'existent sous forme de statuts déontiques qu'en étant attribuées, explicitement ou implicitement, par les autres participants à la pratique discursive. Il faut ici insister sur le fait que Brandom (2010, p. 223) ne privilégie pas l'étude des inférences dont la correction relève de la seule forme logique, mais adhère pleinement à l'idée de Sellars (1953) selon laquelle les inférences matérielles ne peuvent être réduites à des inférences formelles, leur validité pouvant ultimement reposer sur des règles matérielles, déterminant des transitions ${ }^{14}$ (non enthymématiques) entre des contenus, transitions qui, d'un point de vue strictement logique, ne seraient pas concluantes. Il s'agit ici d'une inférence sémantique au sens «matériel» du terme, c'est-à-dire qui résulte de la signification des concepts impliqués et utilisés dans un langage naturel (Brandom, 2010, p. 229). Par exemple, de «je

13 Voir Brandom (1983, p. 644) : «The meanings do not determine the appropriate inferences but what inferences are socially appropriate determines the meanings of the sentence involved in those sentences».

14 Voir Brandom (2010, p. 223) : «Les inférences matérielles [...] sont comprises de manière privative : comme des enthymèmes qui résultent de l'occultation ou du masquage de l'une des prémisses requises pour disposer d'une véritable garantie». 


\section{Dialogue}

vois un éclair maintenant», il est possible de déduire «j'entendrai bientôt le tonnerre». La validité des inférences dépend ainsi du sens des mots ou concepts utilisés, comme «éclair» et «tonnerre». Comprendre ces termes, c'est posséder ipso facto une maîtrise pratique de ces inférences, inférences qui n'impliquent pas seulement «des relations entre contenus propositionnels», mais aussi potentiellement «des relations entre les différents interlocuteurs» (Brandom, 2011, p. 883). C'est donc en fonction de cette approche sémantique, conçue sous la forme d'une explicitation de l'articulation inférentielle, que Brandom élabore son modèle de l'usage du langage comme jeu de marquage au score structuré par des actes de langage qui engagent leurs auteurs dans un espace d'interlocution où les étapes de l'argumentation sont tenues sous la forme d'une archive des «coups» joués par les différents interlocuteurs (ibid., chap. 3, section IV). Autrement dit, la signification des termes et des concepts est construite à partir de ce jeu normatif des interlocuteurs qui se comprennent comme engagés par des raisons (Brandom, 2011, p. 512) et comme possédant certaines permissions vis-à-vis des assertions de leurs partenaires. Ce concept de «marquage au score» est un concept provenant du baseball. Concrètement, c'est la manière dont nous comptons le score (c'est-à-dire les points marqués) au baseball. Brandom emprunte le concept à son maître, David Lewis (1979). Néanmoins, il en modifie l'usage. Pour Lewis, il s'agit d'analyser «les éléments du score conversationnel», c'est-à-dire les «présupposés» déterminant «l'acceptabilité de l'énonciation d'une phrase particulière» (Brandom, 2010, p. 356). Or, là où Lewis pense ces présupposés comme des «usages mentaux», Brandom comprend les «états mentaux tels que la croyance tout autant que leurs contenus représentationnels [...] en référence au rôle qu'ils jouent dans les pratiques de marquage au score, plutôt que l'inverse» (ibid., p. 364). Ce concept prend son sens dans la logique de la sémantique inférentielle : si nos assertions ont un contenu propositionnel, c'est «en référence aux pratiques consistant à donner et à demander des raisons» (ibid, p. 286). Le marquage au score consiste ainsi à compter non plus les points du baseball, mais quelles assertions sont appropriées et quelles différences elles introduisent dans le score total du réquisit de justification de la sémantique inférentielle (ibid, p. 287). Grâce au modèle du jeu de baseball, Brandom explicite comment fonder une sémantique inférentielle — dont les contenus conceptuels comptent comme des raisons (compter un score) - sur une pragmatique normative (jouer au baseball). Aussi Brandom fait-il sienne l'explication épistémique de la signification proposée par Dummett (1981, p. 295-363), selon laquelle le contenu de l'assertion est déterminé par les permissions et engagements dont il découle et par les permissions, les engagements et les interdictions qu'il produit ${ }^{15}$. Plus précisément, il s'agit de distinguer les circonstances appropriées qui nous engageraient à appliquer l'expression de celles qui nous

15 Voir Brandom (2009, p. 56) : «Saisir ou comprendre un tel concept, c'est avoir une maîtrise pratique sur les inférences dans lesquelles il est impliqué [...] [et] être 
permettraient d'appliquer l'expression, et de distinguer les conséquences envers lesquelles nous sommes engagés en appliquant ou en employant l'expression des conséquences envers lesquelles nous gagnons ou perdons une permission d'engagement en appliquant ou en utilisant l'expression. Le contenu envers lequel un locuteur est engagé en assertant $p$ est déterminé par les assertions ${ }^{16}$ qu'il peut faire pour justifier son engagement envers $p$ (et qui portent sur les circonstances d'application de $p$ ), et par les assertions envers lesquelles il s'engage et qu'il a ou non la permission de produire à la suite de son engagement envers $p$ (conséquences de $p$ ). Ces circonstances et conséquences peuvent être d'autres contenus, mais aussi d'autres paramètres non linguistiques : les premières visent à relever le pointage des conversations dans lesquelles il est approprié et légitime d'énoncer l'acte de discours en question; les secondes retranscrivent et suivent l'évolution du pointage ${ }^{17}$ — en termes de transformations des attitudes déontiques par lesquelles les interlocuteurs tiennent les comptes des engagements et permissions des autres — que l'énonciation légitime de ce même acte de discours implique. La pratique linguistique peut alors être comprise comme une fonction déterminant la façon dont le pointage déontique impose, à chaque étape d'une conversation, d'identifier les performances qui sont appropriées ainsi que les conséquences qui suivent des différentes performances c'est-à-dire la façon dont celles-ci modifient le pointage (Brandom, 2010, p. 357). Il est, par conséquent, important de connaître les contenus qui peuvent justifier l'assertion, et non seulement ceux qui en découlent. Le contenu se définit ainsi à partir de son rôle de prémisse, mais aussi de conclusion dans un raisonnement. Comprendre ce que nous disons, ou encore ce que notre interlocuteur dit, consiste à être apte à déterminer ce qui suit d'une assertion, ce qui la prouverait et ce qui la réfuterait, les conséquences envers lesquelles nous nous engageons en la produisant, et les raisons qui justifieraient notre engagement (Brandom, 2002, p. 95; 2009, p. 101). Nous ne pouvons donc exprimer, juger ou asserter un énoncé sans le comprendre et sans l'endosser. Le comprendre, c'est savoir à quoi nous nous engageons en le formulant, ce qui s'ensuit et ce qui peut s'ensuivre. L'endosser, c'est ainsi s'engager envers ce qui est dit : être prêt à le défendre et à se porter garant de son autorité (Brandom, 2002, p. 360).

(suite)

capable de distinguer [...] ce qui découle de l'applicabilité d'un concept et ce dont il découle».

MacFarlane rend compte du rôle de l'assertion selon les quatre catégories suivantes : «1. To assert is to express an attitude; 2 . To assert is to make a move defined by its constitutive rules; 3 . To assert is to propose to add information to the conversational common; 4. To assert is to undertake a commitment» (2011, p. 80).

17 En faisant le parallèle avec ce jeu de pointage au baseball, Brandom parlera par exemple du statut que peut avoir une performance en tant que balle, prise ou retrait (voir 2010, p. 357). 
Si, par exemple, nous affirmons que nous sommes souffrants, nous permettons à autrui de penser que nous souffrons et nous sommes obligés de reconnaître que nous éprouvons de la douleur (Brandom, 2010, p. 317-320). Asserter que $p$ («il pleuvra demain»), c'est, d'un côté, accepter la responsabilité de montrer que nous avons la permission d'avoir cet engagement envers $p$ (notamment en produisant de nouvelles assertions ${ }^{18}$, et donc de nouveaux engagements) et, de l'autre, se porter garant de la vérité de $p$, en permettant aux autres locuteurs de s'engager envers $p$ et envers ses conséquences déductives («les rues seront humides» [Brandom, 2010, p. 219]) en les déchargeant de la responsabilité de prouver que $p$, ces derniers pouvant se déférer ${ }^{19}$ au locuteur initial. Nos assertions ne peuvent donc avoir de contenu que parce que nous sommes engagés dans ce jeu continu de production et de demande de raisons (Brandom 2010, chap. 3) — nommé ci-après $\mathrm{JPDR}^{20}$ — dans lequel nous enregistrons les points des

18 Voir Brandom (1983, p. 641) : «In asserting a claim one not only authorizes further assertions, but commits oneself to vindicate the original claim, showing that one is entitled to make it. Failure to defend one's entitlement to an assertion voids its social significance as inferential warrant for further assertions».

19 Voir Brandom (2010, p. 344) : «La seconde manière de justifier un engagement en prouvant qu'on y est habilité est d'en appeler à l'autorité d'un autre émetteur d'assertion. [...] [Les locuteurs] peuvent invoquer l'autorisation ou l'autorité de l'émetteur, s'en remettant ainsi à l'interlocuteur qui leur a communiqué cette affirmation, et déléguant à cet autre individu toute demande de preuve d'habilitation. [...] Le fait qu'une assertion formulée par $A$ ait, du point de vue social, pour portée d'autoriser une réassertion par $B$ consiste dans le fait qu'il est convenable que $B$ défère à $A$ la responsabilité de prouver l'habilitation à cette affirmation».

L'expression «jeux de production et de demande de raisons» vient de Brandom, qui l'attribue à Sellars (Brandom, 2009, p. 200), mais seulement un passage chez Sellars semble confirmer ce point. Cette expression y apparaît dans des termes proches, tels que «[...] l'espace logique des raisons, des justifications et des aptitudes à justifier ce que l'on affirme» (Sellars, 1956, §36). De son côté, Dummett en vient presque à la conceptualiser en écrivant : «The process of learning to make assertions, and to understand those of others, involves learning what grounds, short of conclusive grounds, are regarded as justifying the making of an assertion, and learning also the procedure of asking for, and giving, the grounds on which an assertion is made» (Dummett, 1981, p. 355). Il emploie l'analogie avec les échecs, en parlant des assertions en termes de «jeu», mais il n'en fait pas une notion centrale de sa sémantique (Dummett, 1978, p. 2; 1981, p. 2 et p. 355). Wittgenstein décrit une fois l'acte d'asserter comme «un coup dans un jeu de langage» (Wittgenstein, 2005, §22). Habermas évoque aussi la notion de jeu de langage argumentatif : "These constitutive rules of the language game of argumentation govern the exchange of arguments and of "yes" or "no" responses [...]» (Habermas, 1998b, p. 45). 
engagements de chaque participant, par le biais de cartes de marquage déontique où nous déterminons ce qui prouverait ou réfuterait de tels engagements, les conséquences envers lesquelles nous nous engageons en les produisant, les raisons qui justifieraient notre engagement (Brandom, 2002, p. 95; 2009; p. 101), ainsi que les points des permissions, c'est-à-dire d'assertions que nous avons défendues avec succès dans ce jeu. Trois caractéristiques propres aux assertions gouvernent ces actions de marquage au score, à savoir : 1) quand un agent formule une assertion, il contracte (par une reconnaissance manifeste et explicite) un engagement doxastique et assume tous les engagements et autorisations qui découlent de l'engagement qu'il reconnaît explicitement; 2) une assertion réussie — dont l'auteur est autorisé à énoncer l'engagement reconnu — autorise d'autres membres de la conversation à prendre le même engagement, à en prendre la responsabilité et à s'engager à son endroit en vue d'une adhésion publique; 3) l'auteur de l'assertion prend la responsabilité de justifier son assertion lorsque les autres membres le lui demandent. Par conséquent, demander des raisons pour une affirmation, c'est en demander les garanties c'est-à-dire ce qui autorise son auteur à prendre cet engagement. Ainsi, le sens de l'assomption qui détermine la force des actes de discours assertifs implique, au moins, une sorte d'engagement à l'égard duquel l'autorisation du locuteur peut toujours être défiée et remise en question (Brandom, 2010, p. 335-339). Cette approche se fonde donc bien sur une théorie des assertions et considère le jeu de dialogue entre deux agents comme essentiel au processus d'offre et de demande de raisons conçu comme une activité de construction de preuves et de réfutations de propositions. De plus, Brandom, influencé par Dummett, exprime la signification des particules logiques en termes de preuve théorique ${ }^{21}$, qu'il retranscrit adéquatement en termes de JPDR. Cependant, Habermas (2001) reproche à ce modèle de communication de ne pas être dialogique au sens étroit où il requerrait un «face à face» (Brandom, 2000a, p. 362) entre un locuteur et un interlocuteur. Nous suggérons donc de concevoir ce JPDR, en termes de règles de particules logiques ${ }^{22}$ et de

21 Cf. au modèle de Dummett dans Brandom (2010, p. 248-251; 2009, p. 69-70). Brandom distingue cependant son inférentialisme du type d' «assertabilisme» que l'on trouve chez Dummett en distinguant deux types principaux de statuts normatifs, «les engagements et les permissions», là où Dummett (1981, p. 188) ne fait appel qu'à un type de statut normatif, c'est-à-dire à un énoncé assertable ou à un locuteur ayant des raisons suffisantes de l'asserter (Brandom, 2009, p. 199). L'interaction entre ces deux types de statuts normatifs génère une sémantique de l'incompatibilité basée sur le fait que «deux contenus assertables sont incompatibles là où l'engagement de l'un exclut l'autorisation à l'égard de l'autre» (Brandom, 2009, p. 204).

22 En logique dialogique, les lignes directrices de tout jeu sémantique ne visent à définir les connecteurs logiques ni en termes de conditions de vérité (Wittgenstein, 2001, 5.101; Church, 1956, p. 37), ni en termes de règles d'introduction et d'élimination 
prétentions à la validité (Habermas, 2001, p. 85-86), comme un ensemble de jeux entre strictement deux personnes - un proposant $P$ et un opposant $O$-, et de voir si le modèle de Brandom répond à cette conception de l'intersubjectivité définie comme strictement dialogique. Une assertion est définie comme un coup dans ces types de jeu et la logique dialogique de Lorenzen ${ }^{23}$ convient parfaitement pour analyser ces jeux d'assertion dans la mesure où «demander des raisons» correspond à des «attaques» en logique dialogique alors que «produire des raisons» correspond à des «défenses». De plus, les «attaques» sont parfois décrites comme des «droits» alors que les «défenses» le sont comme des «devoirs ou des obligations» (Lorenz, 1981, p. 120). Mathieu Marion le résume ainsi : «rights to attack $\Leftrightarrow$ asking for reasons; duty to defend $\Leftrightarrow$ giving reasons» (2012, p. 148). Habermas décrit également la pratique argumentative ${ }^{24}$ dans les termes d'une «compétition coopérative» entre un proposant qui, en tant qu'il «avance une prétention à la vérité[,] est obligé de disposer de justifications» et

\section{(suite)}

de connecteurs logiques (Gentzen, 1935, 1969, §5.13, p. 80), mais en termes de règles de jeu entre deux personnes - entre un proposant $P$ et un opposant $O$-, et à définir la validité logique en termes de l'existence d'une stratégie gagnante pour $P$. C'est-à-dire que, pour tous les coups que peut choisir $O, P$ doit avoir à sa disposition au moins un coup possible afin de remporter la victoire.

Dans des termes informels, les jeux de Paul Lorenzen (voir Lorenzen et Lorenz, 1978) commencent avec le proposant $P$ énonçant une assertion, l'opposant $O$ le mettant au défi de la justifier, et les deux interlocuteurs jouant à tour de rôle. $P$ étant le défenseur et $O$ l'attaquant, les règles qui définissent la signification des connecteurs sont les suivantes : quand $P$ asserte A \& B, $O$ choisit l'un des conjoints et $P$ doit le défendre, et le jeu continue pour ce conjoint-ci; quand $P$ asserte A V B, $O$ demande alors que $P$ choisisse et défende l'un des deux disjoints; pour une implication $\mathrm{A} \Rightarrow \mathrm{B}$, $O$ n'a pas d'autre choix que de concéder A afin d'obliger $P$ à défendre $\mathrm{B}$; et quand $P$ asserte une négation $\neg$ A, $O$ n'a pas d'autre choix que d'asserter A, mais les rôles (attaquer ou défendre) sont alors inversés dans la mesure où $O$ doit maintenant défendre A contre $P$. Pour les quantificateurs, quand $P$ asserte $\operatorname{VxF}(\mathrm{x}), O$ choisit une valeur pour $x$ et $P$ doit alors montrer que $x$ a la propriété $F$, et quand $P$ asserte xF(x), alors $O$ demande que $P$ produise un $x$ qui ait la propriété $F$. Pour une introduction en français, voir Fontaine et Redmond (2008); pour une formulation plus formelle, voir Rahman et Keiff (2005).

Voir Habermas, 1998b, p. 43-44 : «I will limit to the observation that we engage in argumentation with the intention of convincing one another of the validity claims that proponents raise for their statements and are ready to defend against opponents». Il décrit le processus dialogique en termes de droits et d'obligations : «The equal distributions of communicative freedoms and the requirement truthfulness in discourse have the status of argumentative duties and rights, not of moral duties and rights» (ibid., p. 44-45). 
un opposant qui, en tant que «celui qui la reçoit, a, quant à lui, le droit de la contester» (Habermas, 2001, p. 90-91). Au moins deux règles clés sont à mentionner, même si notre but n'est pas de discuter des règles structurelles : la première — la règle du gagnant — énonce qu'un tel jeu prendra fin dans un nombre fini d'étapes dialogiques ou de coups et soit $P$, soit $O$ gagnera si et seulement si le dernier joueur ne peut plus émettre aucun coup ${ }^{25}$; la seconde — la règle formelle pose que $P$ n'a pas le droit d'introduire de formules atomiques si ce n'est celles que $O$ a déjà utilisées et établies au préalable. Pour gagner ou réussir à défendre avec succès nos assertions contre un opposant, nous devons être capables de les appuyer d'une justification ou d'en fournir les raisons. La notion centrale est donc celle de jeu. En nous limitant à un niveau strictement philosophique, nous devons reconnaître le fait que, contrairement aux systèmes dialogiques, qui mobilisent deux joueurs, les systèmes de déduction naturelle sont monologiques dans la mesure où une seule personne est impliquée (Marion, 2012, p. 148). Rappelons que le but de la règle formelle est que tous les éléments nécessaires pour permettre à $P$ de prouver la validité de l'assertion qu'il attribue à $O$, doivent au préalable être concédés par $O$. Le contraste entre le raisonnement monologique et le raisonnement dialogique se retrouve chez Brandom (2011, p. 883-884 et p. 1040), mais, il y est construit différemment :

Les contenus conceptuels employés dans le raisonnement monologique, raisonnement où toutes les prémisses et toutes les conclusions sont des engagements potentiels d'un individu, se greffent sur, et ne sont intelligibles qu'en référence aux contenus conceptuels conférés par le raisonnement dialogique, dans lequel la question de savoir ce qui suit de quelque chose implique essentiellement des évaluations à partir des différentes perspectives sociales d'interlocuteurs ayant des engagements d'arrière-plan différents (ibid., p. 883-884).

Brandom évoque le processus conversationnel sur le mode interpersonnel du «je-tu» où chaque participant doit pouvoir entrevoir le monde à partir de la perspective d'autrui (ibid., p. 1052-1058), mais il ne le lie pas à la sémantique de la même manière qu'on peut le voir ici avec la logique dialogique. Or, il ne quitte pas le cadre de la déduction naturelle (Brandom, 2010, p. 248-251; Brandom, 2009, p. 69-70; voir Trafford, 2017, p. 49). Dans sa réponse à Habermas, Brandom $^{26}$ concède ce point à Habermas qui, de son côté, soutient que

25 Cette règle peut s'énoncer ainsi : le jeu est fini lorsque le dernier joueur ne peut plus jouer; si le dialogue est fermé, alors le proposant gagne, si le dialogue est ouvert alors l'opposant gagne. Ici, «fermé» veut dire que la même formule revient deux fois, assertée par $O$ et par $P$; si ce n'est pas le cas, le dialogue est «ouvert».

26 Brandom (2000a, p. 362) : «I think this is on the whole a fair characterization. There are, to be sure, some elements that are not merely observational in the pragmatics we might think of the fundamental notion of a challenge, which supports the 
Brandom ne rend pas «sérieusement justice au rôle spécifique de la deuxième personne» (Habermas, 2001, p. 112). Brandom admet certes que, dans son modèle, la référence au rôle de la seconde personne repose sur l'attitude logique et observationnelle du rôle d'interprète de la troisième personne ${ }^{27}$, mais il n'y voit aucun conflit. En effet, ce sont bien, selon lui, les agents directement impliqués dans la conversation, et non un arbitre externe, qui évaluent la fiabilité des exigences de justification et les engagements assertionnels auxquels nous sommes ou non autorisés (Brandom, 2009, p. 204). L'accent est mis sur la relation bipolaire «je-tu» comme lieu de production des standards normatifs et n'accorde pas de statut privilégié au «nous» de la communauté. Il sera donc question d'examiner si cette lacune présumée d'une dimension dialogique constitue une entrave au processus discursif de la communication.

\section{Objections et réponse de Habermas}

Selon Habermas, Brandom ne réussit pas à relier sa théorie de l'assertion à la perspective sociale sur le mode du «je-tu» et en reste à une perspective sur le mode du «je-ilı» ${ }^{28}$, où le rôle conféré à une troisième personne est celui d'observateur ou de spectateur et non d'interlocuteur :

Ce n'est pas un hasard si Brandom préfère identifier l'interprète à un public qui apprécie l'acte de discours d'un locuteur, et non à un destinataire dont on attend qu'il réponde au locuteur. Chaque round d'une nouvelle discussion s'ouvre sur une

(suite)

characterization of discursive practice as a "game of giving and asking for reasons". And on the semantic side, the symmetry of the relation between the one acknowledging a commitment $[\ldots]$ and the one attributing it is a deep-seated feature of the account of scorekeeping, communication, and the perspectival character of propositional and other sorts of conceptual content».

27 Le rôle de la troisième personne ne se limite pas, selon Brandom, à celui de spectateur, mais est bien celui d'un acteur participant au processus discursif : «we cannot understand our capacity to understand things unless we take full account of the fact that we are not just spectators trying to conform things, but agents, trying to transform them» (Brandom, 2000a, p. 357).

28 Une remarque de Brandom (2011, p. 1053-1055) nous pousse à croire qu'il s'appuie sur la «triangulation» de Donald Davidson (2001, pp. 119-121, 128-129, 202-203 et 212-213), qui requiert seulement deux locuteurs. Nous notons un point de recoupement, mais sans toutefois que celui-ci soit parfaitement identique à l'intersubjectivité comprise sur le mode du «je-tu». Une série de questions sousjacentes, que nous ne voulons pas traiter ici, est soulevé par Davidson, qui soutient que la triangulation est une condition nécessaire (ibid., p. 128-129) et suffisante (ibid., p. 105) pour l'émergence de la pensée. 
imputation que l'interprète fait du point de vue de l'observateur, point de vue qui est celui d'une troisième personne (2001, p. 113).

Au cœur de cette critique, s'exprime l'idée que la construction sur le mode du «je-tu» de Brandom ne révèle pas, selon Habermas, un authentique «tu» envers lequel le «je» adresse des prétentions à la validité et duquel le «je» attend une réponse, mais davantage une relation «entre une première personne qui élève des prétentions à la validité et une troisième personne qui impute à l'autre des prétentions du même type» (ibid., p. 113). En effet, les interlocuteurs, dans une pratique discursive, n'attribuent ou ne contractent pas seulement des engagements mutuels, mais énoncent des prétentions à la validité en prenant publiquement des positions justifiées par un «oui» ou par un «non» (ibid., p. 115). Le déplacement d'une relation sur le mode du «je-tu» vers une relation sur le mode du «je-il» implique, pour Habermas, que Brandom ne confond pas seulement deux niveaux distincts de communication, mais «ignore aussi le rôle grammatical de la seconde personne» (ibid., p. 114). Brandom néglige alors l'entrecroisement complexe entre la perspective de la première, de la seconde et de la troisième personne, ce qui signifie, toujours selon Habermas, qu'il opère avec un concept inadéquat de la communication en adoptant, de surcroît, une approche méthodologique objectiviste (ibid., p. 106) et individualiste (ibid., p. 116) qui ne convient pas à l'analyse de la pratique discursive. Le dernier mot est donné à un spectateur ou à un observateur qui attribue des engagements et des autorisations à autrui en les évaluant et en les contractant lui-même, rendant de ce fait possible une description objectiviste ${ }^{29}$ de la pratique discursive. Le spectateur ou l'observateur joue le rôle d'un théoricien qui conçoit la compréhension d'une expression comme «une opération d'imputation» (ibid., p. 114) de son interprétation et de son évaluation orientée vers le locuteur $^{30}$. Finalement, dans le modèle de marquage au score de Brandom, la pratique discursive naît davantage sur «fond d'observations réciproques, d'inférences auxquelles procèdent les participants individuels, chacun pour

29 Pour Habermas, Brandom opère à la fois par le biais d'un réalisme conceptuel et d'un idéalisme linguistique objectif «qui n'accorde à l'expérience qu'un rôle de médiateur passif» (2001, p. 106). Rorty adresse une critique similaire à Brandom : «I think that [Brandom's claim], "If we had never existed [...] there would have been facts (truths) going unexpressed" is at odds with another claim Brandom makes : that the "notion of a fact (true claimable) is only intelligible relative to that of a vocabulary". Brandom denies that the second claim makes trouble for the first» (Rorty, 2000b, p. 184).

30 Habermas mentionne que «Brandom part de l'idée que, dans le cas élémentaire, le résultat de la communication consiste dans une relation épistémique, à savoir entre le propos que le locuteur tient sur quelque chose qui existe sur le monde et l'imputation de ce propos à laquelle procède l'interprète» (2001, p. 115). 
soi» (ibid., p. 116) que comme le résultat d'un travail coopératif partagé. Nous pourrions contracter des engagements, mais nous ne pourrions jamais vraiment les partager dans la mesure où nous entretenons des croyances différentes ou reconnaissons des engagements différents (Scharp, 2003, p. 45-46). Habermas (2001, p.114) exigerait une forme plus robuste d'interaction pour satisfaire à «l'impératif de l'intégration sociale» ${ }^{31}$, celle-ci ne demandant pas seulement de prendre position, mais bien «la nécessité de coordonner les plans d'action des participants» (ibid, p. 115) afin de reconnaître «une même prétention à la validité et [de] partager un savoir au sens strict» (ibid., p.116).

Si nous suivons Habermas, il faudrait substituer l'intersubjectivisme comme correctif à l'objectivisme présumé de Brandom. Habermas critique Brandom en se plaçant du point de vue de l'interaction, c'est-à-dire du point de vue d'une théorie du dialogue. Plutôt que d'adopter une position intersubjectiviste discursive, il avance un intersubjectivisme dialogique et interactionniste (Strydom, 2006) se concentrant sur la stricte relation entre une première et une seconde personne. D'un côté, une première personne est un locuteur qui associe une intention à un acte de discours et qui, comme interlocuteur, adresse cette assertion à un destinataire en lui demandant de répondre publiquement par l'affirmative ou par la négative. Une telle réponse est nécessaire soit pour confirmer, soit pour corriger et réviser des opinions sur lesquelles les deux parties doivent être en mesure de s'appuyer lors de leurs interactions futures (Habermas, 2001, p. 115). De l'autre, il ne peut y avoir de deuxième personne sans qu'une première personne la reconnaisse comme son interlocuteur. Habermas propose ainsi d'adopter comme paradigme la relation entre un locuteur et un interlocuteur en la dissociant du statut de la troisième personne, limité au rôle passif d'observateur ou d'interprète qui compte les points des locuteurs engagés. Contre la tendance de Brandom à concevoir la communication ou la pratique discursive calquée sur les modèles du baseball, des procédures judiciaires ou de la danse de salon - comme un processus intelligible sur un mode parfaitement autonome généré à partir d'observations réciproques et d'inférences auxquelles procèdent les contributions individuelles, Habermas formule le premier principe de sa théorie de l'intersubjectivité (2001, p. 113-117). La communication ou la pratique discursive est un processus coopératif d'apprentissage qui se déploie, d'une part, au sein des limites de l'environnement social et sur un contexte de

31 Cet impératif, à la base de l'agir communicationnel, se retrouve auparavant chez Habermas : «De façon générale, coordonner l'action sert à garantir l'intégration sociale d'un monde vécu partagé sur le mode de l'intersubjectivité par les participants» (1988, p. 247; notons que le texte de 1988 est repris en 2018 dans Parcours 1 [Habermas, 2018]). James Swindal souligne aussi : «So while Brandom sees communication as a self-sufficient process of informing, Habermas views it as guided by the imperative of social integration - rational coordination of action plans» (Swindal, 2007, p. 118). 
présuppositions pragmatiques $^{32}$ d'arrière-plan partagées, et qui, d'autre part, est guidé par une communauté de justification visant un accord obtenu par la discussion (ibid., p. 117). La spécificité de la communication, qui, selon Habermas, n'est pas analysée adéquatement par Brandom, repose essentiellement sur la coordination et l'intégration sociale (ibid., p. 115). Est alors centrale à cette conception intersubjectiviste de la pratique discursive la possibilité que les membres d'une communauté emploient les expressions linguistiques en y associant la même signification (Habermas, 1996, p. 4 et p. 9; 1986, p. 205) et qu'ils en viennent ainsi à partager un savoir ${ }^{33}$ (Habermas, 2001, p. 116). C'est donc sur ce fondement que s'appuie, selon Habermas (ibid., p. 117), la justification intersubjective d'une prétention à la validité en privilégiant la perspective du «nous» ${ }^{34}$ de la communauté.

\section{Critiques de la position de Habermas et réponses de Brandom}

Habermas caractérise l'approche de Brandom (2011) dans son ensemble comme une élaboration novatrice, minutieuse et rigoureusement construite d'un programme de pragmatique formelle sur lequel il travaille lui-même depuis les années 1970 et qui vise, selon lui, à reconstruire les règles universelles du fonctionnement de la communication et les conditions de l'intercompréhension à partir des interactions sociales et communicationnelles. Partageant une approche pragmatique, Habermas adhère pleinement à l'intérêt de Brandom pour les pratiques sociales et linguistiques sur fond desquelles les membres de la communauté, capables de discours et d'action, sont engagés. La particularité de la conception de Brandom (2011, p. 286) est précisément ce

32 Voir Habermas (2005, p. 122-123).

33 Voir Habermas (1976, p. 23) : «The goal of coming to an understanding is to bring about an agreement that terminates in the intersubjective mutuality of reciprocal understanding, shared knowledge [...]. We can see that the word understanding is ambiguous. In its minimal meaning it indicates that two subjects understand a linguistic expression in the same way; its maximal meaning is that between the two there exists an accord concerning the rightness of an utterance in relation to a mutually recognized normative background. In addition, two participants in communication can come to an understanding about something in the world, and they can make their intentions understandable to one another».

34 Brandom critique Habermas précisément sur ce point : «les explications je-nous postulent à tort l'existence d'une perspective privilégiée - celle du «nous», ou de la communauté. La correction objective des affirmations (leur vérité) et de l'application de concepts est identifiée à ce à quoi on adhère à partir de ce point de vue privilégié. L'identification de l'objectivité à l'intersubjectivité comprise de cette façon est fautive en ce qu'elle ne laisse aucune place à la possibilité de l'erreur concernant cette perspective privilégiée; ce que la communauté tient pour correct est correct» (2011, p. 1055). 
modèle de la pratique discursive en termes de JPDR où les participants marquent le score de leurs propres engagements et habilitations ainsi que de ceux des autres. Cependant, Habermas (2001) semble, à première vue, manquer de précision dans sa conceptualisation de l'intersubjectivité en traitant, sans distinction, de la pratique discursive, de l'argumentation, de l'échange communicatif, de la communication linguistique, de la communication quotidienne et de l'interaction. Ce flou conceptuel souligne la nécessité de rappeler la distinction théorique entre deux niveaux de communication sur laquelle repose la position de Habermas depuis son tournant vers la théorie de l'agir communicationnel, à savoir : la distinction entre «l'activité communicationnelle» (ou l'interaction) et la «discussion». Habermas l'exprime ainsi :

Dans l'activité communicationnelle, la validité des énonciations est naïvement présupposée afin de permettre l'échange d'informations (d'expériences se rapportant à l'action); dans la discussion, sans échanger des informations, on soumet à discussion des prétentions à la validité problématisées ${ }^{35}$.

La discussion est ici perçue comme une forme réflexive de communication qui, contrairement à la communication ordinaire et quotidienne, transforme, en les soumettant à l'examen réflexif et critique, toutes prétentions à la validité implicites, acceptées jusque-là comme autant d'évidences intersubjectivement partagées, en énoncés hypothétiques et controversés. En effet, dans l'activité communicationnelle, si un acte de discours suscite un doute, le locuteur peut immédiatement tenter de le dissiper, directement dans le contexte d'action. Toutefois, si le doute persiste à la suite d'une telle justification immédiate, c'est que le consensus d'arrière-plan à propos des prétentions à la validité ne suffit plus à couvrir le bon fonctionnement de la pratique communicationnelle et que celles-ci doivent être justifiées explicitement. Nous nous trouvons alors devant la nécessité de quitter la pratique quotidienne de la communication pour passer à un autre niveau de communication, celui de la discussion, qui représente une forme réflexive de l'activité communicationnelle dans laquelle les prétentions à la validité faisant l'objet d'un litige doivent faire leur preuve pour déterminer si elles peuvent ou non être honorées et susciter la recherche coopérative d'un consensus. Cette distinction entre l'interaction et la discussion est fondamentale pour comprendre la nature de la critique de Habermas.

En effet, en critiquant Brandom du point de vue de l'interaction, Habermas (2001) réduit ici la communication à son premier niveau strictement dialogique et, ce faisant, tend à ignorer le second niveau réflexif de la pratique discursive,

35 Habermas, «Conférences Christian-Gauss. Contribution à la fondation de la sociologie sur une théorie du langage. Cinquième conférence» (1971, p. 152). La conférence est reprise dans Parcours 1 (voir Habermas, 2018). Nous ajoutons les italiques dans la reprise des deux concepts. 
centrale au JPDR (Brandom, 2009, p. 204). Il n'y aurait pas lieu de critiquer cette approche s'il était seulement question de la communication ordinaire, mais l'enjeu dépasse ici la sphère de l'interaction ou du dialogue. L'approche dialogique de Habermas envisage la finalité sociale de l'entente comme le résultat de la coordination des significations des actions communicatives de chaque participant orientées vers une compréhension mutuelle ${ }^{36}$, celles-ci étant herméneutiquement interprétées et comprises par les autres participants (Habermas, 2001, p. 117). Cependant, Brandom rejette cette prémisse (Brandom, 2000a, p. 363) selon laquelle le but de la communication est l'atteinte d'une compréhension mutuelle ${ }^{37}$, en soulignant son caractère partiel et incomplet qui ne rend pas adéquatement compte de l'essence même du processus de compréhension, celui-ci devant davantage être perçu comme le produit d'une coordination partagée de différentes perspectives individuelles contribuant, à l'image des pions dans un échiquier, à faire avancer ce JPDR. Brandom exprime ce point au moyen de la métaphore des danseuses de salon $^{38}$ :

36 Voir Brandom (2000a, p. 363) : «From his point of view, the achievement of this sort of mutual understanding is the central communicative phenomenon [...]. Perhaps so. But I am not so convinced. To begin with, mutual understanding in the strong sense Habermas is insisting upon is not required for the undertaking of joint projects».

37 Habermas développe, dans ce sens, une pragmatique universelle qui définit la communication comme une action orientée vers une comprehension mutuelle : «The speaker must have the intention of communicating a true proposition [...] so that the hearer can share the knowledge of the speaker» (1976, p. 22). Le locuteur s'engage alors à fournir les raisons justifiant l'acceptabilité de son assertion : «On comprend une proposition énonciative lorsqu'on sait les raisons qu'un locuteur devrait invoquer pour convaincre un auditeur de son droit d'élever une prétention à la vérité pour telle ou telle proposition. Les conditions de compréhension [...] dans la pratique quotidienne de la communication, renvoient donc à la présupposition d'un jeu d'argumentation dans le cadre duquel le locuteur, en tant que proposant, est en situation de convaincre un auditeur, en tant qu'opposant, du bien-fondé d'une prétention à la validité éventuellement problématique» (Habermas 1988, p. 295).

38 Voir Strydom (2006, p. 168, n. 1) : «[...] that Pierre Bourdieu (1990, p. 59, n. 8) analysis of dance as a model of social integration involving the "synchronization of the homogeneous and orchestration of the heterogeneous" indirectly supports Brandom's example of Fred Astaire and Ginger Rogers dancing to illustrate how different things are being done at the same time as the participants are engaged in a shared practice [...]. Where Bourdieu and, I think, Brandom see a shared situational structure or, rather, a structuring practice which allows participants nevertheless to execute different yet coordinated actions, Habermas (2000, p. 347; 2001, p. 117) insists that the participants are at best engaged in "mutual observation" and that 
The participants do not need all to be doing the same thing (sharing) in a narrow sense in order to be engaged in a joint enterprise, and in the broader sense to be doing the same thing (sharing) [...]. Conversational partners should not be pictured as marching in step, like soldiers on parade, but more as ballrooms dancers, each making different movements (at any moment, one leads and the other follows [...] and so on) and thereby sharing a dance that is constituted by the coordination of their individually different movements (2000a, p. 363).

L'objectif poursuivi par Brandom (2011, p. 1050-1051) est avant tout de décrire une pratique assertionnelle plus fondamentale qui puisse rendre compte de cette différence, au cœur de la structure sur le mode interpersonnel du «je-tu», entre les engagements et autorisations reconnus par le locuteur (c'est-à-dire ceux qui sont attribués par le marqueur) et ceux assumés ou endossés par le marqueur, qui génère cette distinction entre ce qui est considéré comme correct dans la manière d'employer un terme ( par le locuteur) et ce qui est objectivement correct (selon le marqueur). Ce envers quoi le locuteur est objectivement engagé est ce qui est rendu explicite dans l'attribution de re. Brandom considère clairement, en le justifiant, que l'objectivité est un produit (relatif) de ces pratiques sociales discursives $^{39}$. La dimension sociale et ses différentes perspectives inférentielles sont moins des limites que des ressources pour reconstruire l'objectivité. Bien que le langage et la pensée nous amènent, à notre insu, à regarder le monde à partir d'une certaine perspective, ce ne sont pas les contenus sémantiques eux-mêmes, mais leurs expressions qui sont propres à une perspective (Brandom, 2009, p. $186-187$; 2011, p. 884). Cependant, en raison de la pluralité des arrièreplans d'engagements inférentiels et doxastiques, deux individus, même en employant les mêmes concepts, n'expriment pas la même chose ou le même engagement doxastique (Brandom, 2009, p. 177). Il n'existe donc pas de manière neutre d'exprimer un contenu conceptuel :

Il est de l'essence des contenus conceptuels d'être expressivement perspectivaux; ils ne peuvent être explicitement spécifiés qu'à partir d'un certain point de vue, sur

(suite)

this excludes "the possibility for them to converge in their intersubjective recognition of the same validity claim and [to] share knowledge in the strict sense of the term"».

Dans ce jeu discursif de pointage, nos engagements doivent être examinés à la lumière des relations d'incompatibilité et c'est seulement s'ils ne sont pas mis en échec par des incompatibilités que nous pouvons continuer à participer au jeu discursif; s'ils sont mis en échec, nous sommes contraints à des réparations afin d'ajuster nos engagements déviants et être de nouveau admis dans le jeu. Brandom sous-entend donc que nos contenus devraient, dans la plupart des cas, converger (2000a, p. 362). 
l'arrière-plan d'un certain répertoire d'engagements discursifs, et la manière correcte de les spécifier varie d'un point de vue discursif à l'autre (Brandom, 2011, p. 1039).

Par exemple, comme le mentionne Brandom (2009, p. 177), il se peut que l'adepte de Zoroastre veuille dire quelque chose d'autre que moi par l'emploi du terme «soleil» en raison de différences dans ses engagements collatéraux (ce mot $\mathrm{a}$, dans sa bouche, une autre signification que celle qu'il a à mon oreille), mais il peut néanmoins parler de la même chose : le soleil en tant qu'astre placé au centre du système solaire. Cela ne signifie pas que nous ne puissions pas néanmoins partager les mêmes contenus. Partager un contenu, c'est précisément être capable, pour chaque individu, de concilier des différences dans la perspective sociale et discursive entre interlocuteurs. Ce perspectivisme est certes inévitable dans l'expression des contenus; néanmoins, il n'entrave pas une communication et une compréhension inter-perspectivales de contenus; celles-ci nécessitent une interprétation permanente, comme capacité à naviguer d'une perspective inférentielle à l'autre sur la base d'une relation instaurée sur le mode interpersonnel du «je-tu» — d'un arrière-plan de valeurs à un autre. En effet, à chaque fois que des contenus propositionnels sont attribués, «il y a un recours implicite à une pratique sociale je-tu dans laquelle un marqueur interprète les performances d'autrui» (Brandom, 2011, p. 1040). S'engager dans une conversation implique ainsi «d'être capable de se déplacer de perspective en perspective, d'apprécier la portée qu'une remarque aurait pour divers interlocuteurs» (ibid., p. 1116). Cependant, Brandom précise que le problème ne s'arrête pas là puisqu'il ne s'agit pas de considérer le marqueur comme le seul garant de l'application correcte et objective des termes. Dans la mesure où la structure du jeu de pointage permet l'itération de marqueurs, un premier marqueur qui reconnaît des engagements et autorisations peut aussi être évalué par un autre marqueur qui effectue lui-même des engagements et autorisations. Cette itération de marqueurs indique précisément une différence entre les engagements que le premier marqueur reconnaît ou est disposé à reconnaître (attitudes déontiques immédiates) comme corrects, et ce envers quoi il est réellement et objectivement engagé (statuts déontiques), sans nécessairement le savoir (ibid., p. 1050-1051) — cette fois-ci selon le point de vue du nouveau marqueur, et ainsi de suite. La procédure de pointage implique la tenue de deux registres de comptes où les points seront comptés pour chaque interlocuteur, mais également par chaque interlocuteur (ibid., p. 361 et p. 870-871) : un premier registre de comptes recueillant les engagements et autorisations que reconnaît l'agent linguistique (les engagements et autorisations attribués par un marqueur au score) et un second registre de comptes indiquant les engagements et autorisations que le marqueur au score assume ou endosse. De là résulte la possibilité d'une différence entre les engagements et autorisations que le locuteur reconnaît et ceux auxquels le marqueur adhère. Cependant, un double livre de pointage peut en retour être tenu sur le premier marqueur par un second marqueur, n'excluant pas encore la possibilité d'une différence 
entre les engagements et autorisations reconnus par le premier marqueur et ceux assumés par le second, et ainsi de suite pour le second marqueur à l'égard d'un troisième marqueur ${ }^{40}$. La structure sociale sur le mode du «je-tu» génère ainsi différentes perspectives de pointage afin de les soumettre à une évaluation comparative $^{41}$ : la compréhension linguistique dépend alors d'une capacité à évaluer sa propre perspective et celle des interlocuteurs, c'est-à-dire en attribuant explicitement ou implicitement des engagements envers des contenus de re ou «en explorant inférentiellement la portée qu'a l'engagement attribué au locuteur dans le contexte des engagements substitutionnels contractés par l'auditoire qui l'attribue» (Brandom, 2011, p. 910). Pour comprendre ce que l'autre dit, nous traduisons ces assertions dans notre réseau d'inférences, à partir de nos pôles référentiels (de re) :

Pour extraire de l'information à partir des remarques des autres, il faut être capable de mettre en œuvre le genre d'interprétation substitutionnelle qui est exprimé explicitement lorsque l'on propose des caractérisations de re des contenus de leurs croyances - il faut, en d'autres termes, être capable de dire ce dont leurs croyances seraient vraies si elles étaient vraies. Il s'agit là d'une saisie du contenu représentationnel de leurs affirmations (ibid., p. 916).

Nous oscillons alors en permanence entre la compréhension d'autrui à partir de nos propres critères, à partir de la manière dont les choses sont pour nous, dans le contexte de nos croyances (de re), et la compréhension d'autrui à partir de ses contenus de croyance (de dicto). Notre capacité à comprendre ce que disent

40 Il faut noter que le jeu discursif n'a pas à être joué jusqu'au bout, mais seulement jusqu'au point où il est plus raisonnable d'accepter une prétention à la validité que de continuer à la défier; dans tout discours ordinaire, ce point vise à être atteint assez tôt. Brandom précise : «Bien souvent, lorsqu'un engagement se voit attribué à un interlocuteur, une habilitation lui est également attribuée, par défaut» (2010, p. 349). Nous explicitons plus en détail dans la section 5 de cet article.

41 Brandom ajoute, en utilisant l'analogie du développement des concepts de la common law, que le processus discursif s'inscrit dans une dimension historique : l'application correcte d'un terme émerge des différences entre, d'une part, nos applications et évaluations actuelles et, d'autre part, celles des autres joueurs de concert avec nos applications, évaluations passées et futures et celles de nos semblables. Les applications passées des concepts exercent une autorité sur les applications futures en fournissant les précédents disponibles pour justifier les décisions ultérieures et, réciproquement, les applications ultérieures de concepts exercent une autorité sur les applications antérieures, le choix du contenu que l'autorité du passé est considérée avoir institué étant décidé par les juges qui rendent aujourd'hui des décisions (2000b, p. 258). Nous ne visons cependant pas à développer davantage ce point ici. 
les autres, au sens où cela nous permet de disposer de leurs remarques et de les employer comme prémisses dans nos propres inférences, dépend donc de cette habileté à spécifier ces contenus en termes de re et non pas uniquement en termes de dicto (ibid., p. 910). Le changement de perspective est ainsi rendu explicite lorsque nous contrastons les attributions de re avec les attributions de dicto $^{42}$.

Par conséquent, l'approche strictement dialogique ne semble pas adéquate pour rendre compte de la spécificité de la pratique discursive comprise comme JPDR où aucune perspective n'est en soi privilégiée ${ }^{43}$ et d'où aucune

42 Brandom prend ainsi l'exemple suivant, qu'il développe à deux reprises (2011, p. 895-901; 2009, p. 186-187) : quand un plaignant $S$ qui, en caractérisant l'affirmation de son adversaire, dit que «l'avocat de la défense $A$ croit que $p$, c'est-à-dire qu'un menteur invétéré est un témoin digne de foi», il attribue (explicitement) à $A$ un engagement doxastique envers $p$, et il reconnaît son propre engagement doxastique envers cet engagement doxastique de $A$ (mais pas nécessairement envers $p$, puisque $S$ peut considérer que $p$ est faux), soit que «l'homme qui vient de témoigner est un menteur invétéré». Si le plaignant était précis dans la caractérisation de l'affirmation de son adversaire, il indiquerait qui est responsable de quoi : l'avocat de la défense affirme qu'un certain homme est un témoin digne de foi alors que le plaignant affirme d'un homme qu'il est un menteur invétéré. Le désaccord concerne la question de savoir si cet homme est un témoin digne de foi, tandis que le plaignant affirme d'un homme qu'il est un menteur invétéré, et non pas de savoir si les menteurs invétérés fournissent des témoignages dignes de foi. L'attribution est alors de re en spécifiant le contenu de la croyance imputée : ce que le plaignant doit dire est «l'avocat de la défense déclare, d'un menteur invétéré, que c'est un témoin digne de foi». Par conséquent, que quelqu'un soit un témoin digne de foi, cela fait partie de l'engagement attribué par le plaignant, et que cet individu soit en fait un menteur invétéré, cela fait partie de l'engagement pris ou contracté par le plaignant. L'attribution est alors de dicto lorsque l'interprète se base uniquement sur des concepts et des liens inférentiels que l'avocat de la défense reconnait, soit «l'avocat de la défense croit que l'homme qui vient de témoigner est un témoin digne de foi». Ainsi, le fait de distinguer, dans le contenu d'une attitude propositionnelle, les attributions qui sont de re et celles qui sont de dicto vise à rendre explicite «la distinction essentielle de marquage au score déontique de perspective sociale entre les engagements attribués et ceux qui sont pris» (ibid., p. 187).

43 Voir Brandom (2011, p. 1056) : «[...] selon l'interprétation je-tu de l'intersubjectivité, chaque perspective est au mieux localement privilégiée en ce sens qu'elle intègre une distinction structurelle entre les applications des concepts objectivement correctes et les applications qui sont seulement subjectivement considérées comme correctes. Ce qui est partagé par toutes les perspectives discursives, c'est le fait qu'il y ait une différence entre ce qui est objectivement correct (dans la façon dont les concepts sont appliqués) et ce qui est seulement considéré 
n'est exclue (Brandom, 2011, p. 1056). Habermas (2001, p. 113) reproche néanmoins à Brandom de ne pas tenir compte de l'entrecroisement complexe qui se produit entre les perspectives de la première, de la deuxième et de la troisième personne. Pourtant, il continue lui-même à réduire cette triple relation sur le mode du «je-tu-il» à une relation sur le mode de la double contingence (Habermas, 1988, p. 280 et p. 301) du «je-tu», en excluant (Habermas, 1996, p. 18) ou en reléguant au rôle d'observateur la perspective d'une troisième personne $^{44}$. Il est pourtant surprenant qu'antérieurement, dans ses conférences Christian Gauss, prononcées en 1971 à l'université de Princeton, il insiste, d'une part, pour redéfinir cette relation entre un alter et un ego, chacun devant être capable de justifier ses actions ${ }^{45}$, et pose les rôles comme étant de ce point de vue interchangeables tout en reconnaissant les différences de perspectives de l'autre comme un autre ego (Habermas, 2018 [1971], p. 104) ${ }^{46}$. D'autre part, il

\section{(suite)}

comme correct, et non la nature de cette différence - c'est la structure et non le contenu». Kevin Scharp (2003, p. 57) précise : «But according to Brandom's theory, one must be able to draw the distinction between taking to be correct and being correct even for the perspective of Brandom's own theory. That is, even Brandom's perspective as theorist cannot be privileged according to his I-thou sociality».

Voir Habermas (1988, p. 276) : «Quant aux énonciations langagières, je les décrirai comme des actes à travers lesquels un locuteur cherche à s'entendre avec un autre sur quelque chose qui existe dans le monde. Ce sont là des descriptions qui conviennent du point de vue de l'acteur, c'est-à-dire du point de vue de la première personne. À cela s'opposent les descriptions faites par une troisième personne, du point de vue d'un sujet observant comment un acteur parvient à une fin [...], ou comment cet acteur s'entend avec quelqu'un, à propos de quelque chose, en formulant un acte de parole. Enfin, du point de vue de la seconde personne, ces descriptions conviennent toujours pour ce qui est des actes de parole [...] et elles conviennent aussi dans le cas des activités finalisées, lorsque cette activité fait partie d'un contexte de coopération».

45 Voir Habermas (1971, p. 153-154) : «Lorsque nous rencontrons un vis-à-vis [...][,] [n]ous ne pouvons entrer avec lui dans une interaction [...] au niveau de l'intersubjectivité, que dans la mesure où nous le présupposons capable de rendre compte de ses actes s'il était interrogé à ce sujet de façon appropriée. [...] Nous procédons donc à une idéalisation, mais qui nous implique, dans la mesure où nous percevons l'autre sujet comme nous percevons nous-mêmes; nous supposons que l'autre [...] est capable de donner des raisons à son action [...] [et] nous sommes convaincus de rendre compte de notre propre action $[\ldots] »$. unequals who are nonetheless aware of their interdependence. Moral universalism must not take - the fact that persons as such are equal to all other persons - at 
fait appel à l'attitude performative ${ }^{47} \mathrm{~d}$ 'une troisième personne — décrite comme «tout partenaire discursif» — ou au jugement des autres ${ }^{48}$ pour reconstruire la structure idéale de la communication (Habermas, 1971, p. 149). Il mentionne également la nécessité d'établir une distribution symétrique des opportunités en termes d'obligations et de permissions ou de droits de tous les participants, sans exclusion, à faire valoir leurs points de vue et à remettre en question ou à critiquer les opinions des autres dans la discussion :

Une telle symétrie de droits et de devoirs peut être assurée par une égalité en termes d'opportunités d'employer les actes de parole régulatifs, autrement dit par une distribution égalitaire des opportunités d'émettre un ordre et de faire opposition, de permettre et d'interdire, de promettre et d'accepter des promesses, de rendre compte et de demander des comptes, etc. Associée à l'emploi à opportunités égales des actes de parole communicatifs, cette condition garantit en même temps la possibilité de sortir à tout moment des contextes d'interaction et de s'engager dans des discussions où sont examinées les prétentions à la validité (Habermas, 1971, p. 151).

Un accord rationnel ne peut donc être réalisé que dans des conditions sociales idéales de liberté et d'égalité ${ }^{49}$ de parole. Si tout énoncé considéré du point de vue de la validité présuppose un locuteur idéal qui prétend obtenir un accord rationnel, et si un accord rationnel présuppose de telles conditions d'égalité et de liberté, alors tout énoncé considéré du point de vue de la validité présupposerait un locuteur qui prétend que de telles conditions sont ou doivent être réalisées. Toute prétention à la validité s'accompagnerait d'une prétention à une situation idéale de parole ${ }^{50}$. Si Habermas va ici au-delà de l'approche dialogique qu'il

(suite)

the expense of the aspect of individuality - the fact that as individuals they are at the same time absolutely different from all others».

47 Nous entendons ici le terme «performatif» ou «le point de vue performatif d'un participant à l'interaction» (Habermas, 2001, p. 85) dans le sens où l'attitude d'une troisième personne est celle d'un acteur impliqué dans la conversation et non d'un observateur ou d'un «auditeur» passif.

48 Voir Habermas (1971, p. 138) : «Pour distinguer les énoncés vrais des énoncés faux, je me réfère au jugement de tous ceux avec lesquels je pourrais chaque fois engager une argumentation (ce faisant, j'inclus tous les interlocuteurs que je pourrai rencontrer si ma biographie personnelle était coextensive de l'histoire de l'humanité)».

49 Cf. Habermas (1987, p. 323-324; 1999, p. 110-111), où il expose en détail les conditions de possibilité d'une discussion rationnelle, ainsi que Habermas (1971, p. 138 sq.; 1987, p. 406-407 et 2001, p. 191), qui résume l'ensemble des conditions, à la fois langagières et non langagières, d'un consensus rationnel.

50 C'est dans la notion de communauté de communication idéale que semble résider l'une des clés de l'articulation entre le concept d'agir communicationnel et le 
offre dans la section $\mathrm{V}(2)$ de son essai sur Brandom, la question du rôle de la troisième personne demeure néanmoins encore en suspens.

Habermas (2001, p. 103-106) propose ainsi de corriger le réalisme conceptuel de Brandom en lui substituant un réalisme pragmatique qui reconnaît la résistance d'un monde objectif indépendant. Cependant, même dans les discours pratiques où le monde social est entrevu depuis la perspective des participants engagés dans la pratique de discussion et de justification, l'attitude de la troisième personne n'intervient que pour décrire naïvement le monde objectif (Habermas 1998b, p. 38). Habermas (1998b, p. 42-43) rend pourtant compte, antérieurement, du processus de délibération et de justification au sein d'un discours conçu sur le modèle d'un processus discursif épistémiquement orienté : les raisons pragmatiques et éthiques exprimées en termes d'orientations et de considération égale des intérêts et des valeurs des participants au discours pratique ne sont pas ignorées, mais généralisées et traitées comme des contributions épistémiques valides subordonnées, certes, aux structures d'un accord intersubjectivement partagé et collectivement établi, mais sans que soit exclue la structure relationnelle du point de vue performatif de l'autre et de la différence. Il n'est donc pas question ici de privilégier la «relation Je-Tu» (Habermas, 2001 , p. 113) et la référence à un monde objectif, mais bien d'inclure les propriétés épistémiques qui sont discursivement construites, en termes de droits et d'obligations argumentatifs (Habermas, 1998b, p. 44-45; 2005, p. 124-125), et collectivement admises, en excluant toute contrainte, hormis celle de la force du meilleur argument (Habermas, 1998b, p.44), dans la mesure où elles sont acceptables du point de vue de la perspective de la troisième personne. Habermas ne semble donc pas se limiter à la position interactionniste qu'il oppose à Brandom en évoquant, du moins entre les lignes, la place de la perspective de la troisième personne dans un tel processus. Par ailleurs, Habermas mentionne que locuteur et interprète doivent renvoyer «au même objet ou au même état de choses» (2001, p. 97) et entretenir des significations identiques afin de garantir la communication ${ }^{51}$. Or, dans son modèle épistémique du discours, une certaine contingence au niveau de la signification semble permise, autorisant les participants à réviser leurs propres interprétations d'eux-mêmes ainsi que celles d'autrui sans que cela n'entrave leur potentielle coordination (Habermas, 1998b, p. 42-43). Néanmoins, il demeure catégorique quant à l'inclusion des perspectives privilégiées de la première et de la deuxième personne (Habermas, 2001, p. 114). Pourtant, dans tout processus communicatif discursif et conjoint, d'autres participants peuvent bien être indirectement impliqués et

\section{(suite)}

concept de démocratie en ce sens que la démocratie pourrait apparaître comme une préfiguration politique concrète d'une situation idéale de parole.

51 Voir Habermas, «Cinq introductions à des monographies non écrites, Tome II. Théorie de la rationalité et théorie du langage» (2009, p. 210). 
leurs rôles s'avérer tout aussi indispensables. Il est par conséquent surprenant de constater le retrait de la perspective de la troisième personne incarné par le rôle et l'autorité épistémique du public, alors que, par ailleurs, Habermas souligne son importance dans la structure interne de la sphère publique où chaque individu ou acteur autonome de la société civile contribue à faire émerger ses principales préoccupations et à les transcrire en termes de questions publiques, traduisant ainsi l'influence de l'opinion publique ${ }^{52}$.

Plus loin dans Vérité et justification, Habermas confirme ce point :

Mais avant d'être considéré comme universel dans l'espace public de la discussion, tout intérêt censé "compter" moralement, en cas de doute, doit être interprété et fondé de façon convaincante, puis traduit en termes de prétention pertinente du point de vue des personnes concernées qui participent à des discussions pratiques (2001, p. 236).

Pourtant, dans la discussion de l'exemple de Brandom (2011, p. 897; 2009, p. 186-187), mettant en scène, dans le cadre de la procédure judiciaire, un procureur général et un avocat de la défense plaidant devant un juge et des jurés quant à la question de savoir si le témoin est un homme digne de foi ou un menteur invétéré, Habermas relègue le juge et les jurés au rang de spectateurs passifs dans l'attente du déroulement du procès :

[...] une telle communication se déroule en fait à deux niveaux. Au premier niveau, l'un et l'autre, le procureur et l'avocat de la défense, parlent l'un avec l'autre en contestant chaque fois l'exactitude de leurs énoncés réciproques [...]. Mais, en même temps, ils ont évidemment conscience de la présence du juge, des jurés et du public qui, à un second niveau de la communication, suivent leur échange et le jugent sans mot dire. Or, significativement, aux yeux de Brandom, c'est la communication indirecte des locuteurs avec un public d'auditeurs, non la communication des personnes directement intéressées, qui est le cas paradigmatique (2001, p. 113-114).

Habermas considère que Brandom dissocie et tient séparément deux niveaux distincts de communication, à savoir un premier niveau de communication qui concerne les acteurs directement impliqués et un second niveau de communication indirecte mettant en scène les locuteurs (les jurés) et les spectateurs (le public) qui se contentent de les écouter. Il apparaît ici que Habermas perd de vue la pratique discursive en tant que telle. En effet, plutôt que d'être tenus séparément, la pratique discursive et le jeu de pointage déontique exigent, au contraire, que ces deux dimensions soient envisagées simultanément. La

52 Voir Habermas (1996, p. 364) : «The public audience possesses final authority because it is constitutive for the internal structure of the public sphere, the only place where actors appear. There can be no public sphere without a public». 
trame de la pratique sociale sur le mode du «je-tu» (et non sur le mode du «je-nous» ${ }^{53}$ ) décrit avant tout, tel que Brandom le démontre, la relation entre un auditoire qui attribue des engagements et qui marque en conséquence les points, et un locuteur qui contracte des engagements, dont la somme des points est établie. L'usage de telles imputations de re et de dicto d'une attitude propositionnelle tourne essentiellement autour des distinctions sociales de perspective doxastique entre celui qui impute un engagement et celui à qui un engagement est imputé - autrement dit, entre le répertoire déontique associé à celui par qui un engagement propositionnel est contracté et celui à qui un engagement propositionnel est par là explicitement attribué. Ainsi, dans le cas discuté, certainement que dans la salle d'audience, les juges qui entendent l'affaire et les jurés qui l'écoutent sont ceux qui suivent de plus près la progression de la discussion et qui tiennent les cartes de marquage au score des parties respectives (c'est-à-dire qui recensent les points marqués par l'avocat de la défense et par le procureur) afin d'être capable d'évaluer la fiabilité et la justesse des propos du témoin en question. Il est certain que la perspective des deux locuteurs (avocat de la défense et procureur général) est plus centrale que le point de vue de l'audience ou encore que la perspective des juges et plus centrale que celle des jurés, mais il ne faut pas pour autant en déduire qu'une perspective prime arbitrairement sur une autre. Brandom veut simplement souligner le fait «qu'une phrase, dans la bouche d'une personne donnée, n'a pas typiquement la même portée que cette même phrase provenant d'une autre personne, même dans le cas de partage intégral du langage et de parfaite compréhension mutuelle» (2011, p. 904). Le succès final de la communication résulte de ce jeu dialogique d'attaque et de défense centré sur la structure sur le mode du «je-tu» et de cette capacité à naviguer à travers les différences de points de vue. La relation sur le mode du «je-tu» entrevue par Brandom est, dans un sens, dialogique, contrairement à la critique de Habermas, dans la mesure où, dit de manière informelle, le locuteur («Je»), en tant que proposant $P$, est en situation de convaincre son interlocuteur («Tu») qui, en tant qu'opposant $O$, doit concéder tous les éléments soutenus par $P$ pour que l'assertion de $P$ soit valide, ce qui correspond au point de vue de la seconde personne (Marion, 2012, p. 149). Il est donc bien question d'un «recours implicite [à] une pratique sociale je-tu dans laquelle un marqueur interprète les performances d'autrui» (Brandom, 2011, p. 1040). Théoriquement parlant, Habermas (1996, p. 139) demeure prisonnier du concept de la double contingence en privilégiant la relation étroitement dialogique du «Je-Tu» au

53 Une réponse à Habermas se lit chez Scharp (2003, p. 56-57). En effet, Brandom (2011, p. 1055) considère qu'il n'y a pas de point de vue «globalement privilégié», ceci incluant aussi celui de la communauté (ibid., p. 1050-1055), alors que Habermas fait la faute d'accorder une perspective privilégiée aux normes de la communauté : il semble ainsi que ce soit la raison qui justifie son interprétation de la théorie de Brandom comme une forme d'«individualisme méthodologique». 
détriment du processus discursif, celui-ci incluant la perspective de la troisième personne.

Pourtant, Habermas conçoit dans Vérité et Justification que pour qu'une vérité soit admise au sein d'une communauté, il faut qu'elle soit reprise, critiquée et défendue dans une argumentation convaincante vis-à-vis d'autres propositions, et suggère la solution d'une discussion rationnelle entre le plus grand nombre de points de vue sans exclusion, la discussion permettant ici de décloisonner les points de vue particuliers et d'accéder à un point de vue impartial sur le modèle des forums scientifiques. Il entrevoit ainsi le discours à l'image d'un jeu de langage décrit tant comme «un forum de discussion» (Habermas, 2003, p. 102) que comme un «processus de construction» discursif conjoint d'un monde partagé (ibid., p. 44 et p. 47) : c'est l'épreuve des vérités déjà établies qu'il faut soumettre à la critique et des autres points de vue qui prétendront eux aussi dire le vrai à propos des mêmes faits ou événements. Les locuteurs n'ont plus d'autre choix que de chercher à s'assurer, par la seule force des bonnes raisons, de la validité de leurs positions respectives, ne disposant d'aucun accès direct à des conditions de vérité qui seraient soustraites au langage et qui pourraient échapper à la médiation interprétative (Habermas, 1992, p. 148-149). Mais pour ce faire, avance Habermas (2001, p. 191), ces locuteurs doivent en même temps admettre des conditions idéales de justification et d'argumentation qui leur permettent de transcender les contextes immédiats de l'action. En effet, ce qui peut être rationnellement considéré comme vrai dans l'espace réflexif du discours doit pouvoir valoir non seulement dans un contexte déterminé, mais dans tous les contextes, en tout temps et devant tout interlocuteur futur. Cela dit, il est bien sûr impossible de justifier une affirmation à la totalité des auditeurs rationnels en tout temps et devant tout interlocuteur futur. Par conséquent, seuls les arguments «ont la force de convaincre ceux qui prennent part à une discussion de la prétention à la validité, autrement dit de motiver rationnellement la reconnaissance des prétentions à la validité» (Habermas, 1978, p. 148-149). Or, cette prétention à la validité d'un savoir est toujours provisoire et peut ultérieurement être remise en question. En ce sens, l'idée d'une acceptation rationnelle est toujours hypothétique et falsifiable, et n'est jamais complètement actualisée dans la pratique. Elle est surtout basée sur cette contrainte de décloisonnement des perspectives des participants au discours ou sur ce travail de distanciation ${ }^{54}$ qu'exige ce jeu de langage décrit comme un «processus d'apprentissage» (Habermas, 2001, p. 102 et p. 108). Or, dans l'approche interactionniste entrevue dans l'essai critique de Brandom, l'identité de signification, l'interprétation herméneutique de

54 Cf. Habermas (2005, p. 125) : «La forme de communication [...] qui est celle de la discussion rationnelle contraint les participants, en les amenant à mobiliser toutes les raisons disponibles et pertinentes, à se décentrer progressivement de leur perspective cognitive». 
la signification des actes de discours et l'évaluation de la compréhension n'accordent aucune place à une telle contrainte de décloisonnement et d'apprentissage discursif.

\section{Apport de Brandom : vers un perspectivisme discursif}

Habermas admet donc la pertinence de cette objection portant sur la prétention à l'universalité. Un énoncé universellement valide est alors un énoncé faisant l'objet d'un accord rationnel universel. Il faudrait donc, pour s'assurer de la validité universelle d'un énoncé, le confronter à tous les êtres rationnels dans l'espace et le temps. Or, dans l'état actuel des choses, tout ce que la procédure dialogique peut obtenir, c'est un accord rationnel «concret», c'est-à-dire un accord obtenu à partir d'un nombre limité d'individus ${ }^{55}$. Or, un accord rationnel concret, même obtenu dans des conditions idéales, n'équivaut pas à un accord rationnel universel. En effet, comme Habermas le mentionne lui-même :

dans la mesure où toutes les discussions réelles, se déroulant dans le temps, sont provinciales par rapport à l'avenir, nous sommes incapables de savoir si les énoncés qui sont aujourd'hui rationnellement acceptables, serait-ce dans des conditions approximativement idéales, résisteront également, à l'avenir, à toute tentative de réfutation (2001, p. 192).

Dans les termes tranchants de Rorty, dire «Je vais essayer de défendre ceci contre tous les opposants» est louable, mais dire «Je peux défendre cela contre tout opposant» n'a pas de sens. Il est impossible de dire que nous pourrons défendre notre position contre tous les contradicteurs, car nous ne savons pas comment seront les futurs contradicteurs ${ }^{56}$. Par conséquent, la prétention discursive permet sans doute de satisfaire à la prétention à la reconnaissance factuelle, mais non à la prétention à l'universalité. Rorty mentionne ainsi que l'idéal d'une vérité objective définie comme accord universel ou prétention à l'acceptation rationnelle universelle n'est pas suffisant pour fournir un cadre contextuel aux participants. La discussion est sans doute une condition nécessaire pour garantir la validité universelle des énoncés, mais non une condition suffisante.

55 Voir Rorty (2000a, p. 56-64 et p. 60) : «Our finitude consists in the fact that there can never be an ideal audience, only more spatially, temporally, and socially restrained audiences».

56 Rorty formule l'exemple du «champion du village» : «It is not the case, as Habermas says, that "What we hold to be true has to be defendable on the basis of good reasons, in all possible contexts." If it were, I would, whenever I acquired a belief, be tacitly making an utterly unjustified empirical prediction about what would happen in a potentially infinite number of justificatory contexts before a potentially infinitely diverse set of audiences. I find this as implausible [...]» (2000a, p. 56-57). 
Plus précisément, si le désaccord de la communauté scientifique garantit que la proposition en question est mal justifiée et donc insuffisante, en revanche un accord, même obtenu dans une situation idéale de parole, ne garantit pas une validité universelle. Cela concerne a fortiori les normes ${ }^{57}$ de la situation idéale de parole. Si celles-ci doivent être établies au moyen de la discussion et si le résultat de la discussion ne garantit pas une validité absolue, alors les conditions idéales de parole ne peuvent être absolument fondées. Rorty (2000a, p. 60) affirme ainsi que ce n'est pas cet impératif d'un accord rationnel universel qui encourage les participants au dialogue à admettre qu'un autre point de vue puisse être meilleur que le leur, mais que c'est bien en se confrontant aux différents points de vue qu'ils reconnaissent que leurs points de vue méritent d'être rectifiés ou réexaminés.

Habermas devrait donc redonner toute sa place au déroulement du processus discursif tel que Brandom le conçoit — sans nécessairement présupposer des normes indépendantes de notre contexte d'usage - , celui-ci reflétant une conception inférentialiste de la pratique discursive en termes de marquage déontique, dans laquelle les marqueurs coopèrent au sein d'une activité conjointe et coordonnent des perspectives sociales en marquant les points selon des pratiques communes. Ce holisme ${ }^{58}$ inférentiel doit ainsi être compris dans le contexte d'un holisme social, et ce holisme social exige que la saisie du contenu sémantique dépende de la capacité des marqueurs à exploiter les relations entre les différentes perspectives constituées par les engagements contractés et attribués à ceux dont ils enregistrent les points déontiques. Chaque marqueur doit donc pouvoir tenir deux registres de comptes afin de suivre le cours de la conversation et recenser les points de chaque engagement et autorisation reconnus par chacun des marqueurs. La communication ${ }^{59}$ s'ordonne ainsi entre deux marqueurs, $A$ et $B$, quand un énoncé $p$ contracté par $A$ devient disponible à $B$ comme prémisse dans le système inférentiel de $B$. Supposons que $B$ détermine que l'énonciation de $A$ a la force d'une assertion et que $B$ comprenne

57 Une discussion est dite rationnelle sous les conditions suivantes : «(a) un déroulement public et l'inclusion complète de tous les intéressés, (b) une égale répartition des droits de communication, (c) le caractère non violent d'une situation n'admettant que la force non coercitive du meilleur argument et (d) la sincérité des déclarations faites par tous les participants» (Habermas, 2001, p. 300; 2005, p. 123). Brandom (2011, p. 205) : «Mais la notion inférentielle de contenu sémantique est essentiellement holiste [...]. On ne peut rien savoir du rôle d'un contenu dans des inférences si on ne sait rien des rôles d'autres contenus dans des inférences, des contenus qui pourraient être inférés à partir de lui, ou desquels il pourrait être inféré». Brandom (2010, p. 369-371). Nous reprenons ici les commentaires de Scharp (2005, p. 211-215). 
le contenu de $p^{60} . B$ doit d'abord attribuer un engagement doxastique à $A$ (qui sera inscrit sous les engagements que $A$ reconnait). $B$ attribue ensuite à $A$ tous les engagements quant aux conséquences inférentielles qui se rapportent à $p$ au titre de conclusion d'inférences préservant l'engagement et ayant $p$ pour prémisse. Il faudrait alors que $B$ ajoute un engagement à toutes les affirmations $q$ qui seraient des conséquences inférentielles et engageantes de $p$ dans le contexte des autres phrases attribués à $A$. Celles-ci varieront en fonction des hypothèses auxiliaires dont on dispose, étant donné les autres engagements que $B$ attribue déjà à $A$. Autrement dit, les engagements qui découlent de $p$ par le biais d'inférences préservant les engagements dépendront des autres engagements que $A$ est considéré comme avoir contracté et reconnu. C'est là ce qui clôt les attributions de $B$ à $A$ sous des inférences préservant l'engagement. Ensuite, $B$ doit examiner les relations d'incompatibilité dans les engagements de $A$ au sein desquelles s'inscrit $p$ (et donc également les conséquences inférentielles engageantes de $p$ ) afin de déterminer lesquelles, parmi les autorisations que $B$ attribue à $A$, sont exclues par le nouvel engagement. Les assertions ajoutent certes de nouveaux engagements; mais, en ce qui a trait aux autorisations, elles peuvent non seulement en ajouter mais aussi en ôter. $B$ doit, par la suite, à la lumière des relations d'incompatibilité associées à tous les engagements attribués à $A$, attribuer des autorisations à toutes les affirmations qui sont des conséquences inférentielles engageantes des engagements auxquels $A$ est déjà considéré comme autorisé, ce qui clôt la somme des points attribués selon les inférences préservant l'engagement, là où les autorisations qui en résultent ne sont pas mises en échec par des incompatibilités. Tout au long de ce processus, $B$ doit donc continuer à vérifier les relations d'incompatibilité afin de s'assurer qu'il n'attribue pas à $A$ des autorisations à deux engagements incompatibles.

La prochaine étape consiste pour $B$ à attribuer des autorisations à toutes les affirmations qui sont des conséquences inférentielles permissives des engagements auxquels $A$ est déjà considéré comme autorisé, ce qui clôt la somme des points attribués selon les inférences préservant l'autorisation (ce que l'on inscrit sous les autorisations que $A$ contracte) qui ne sont pas mises en échec par ces incompatibilités. Il est néanmoins important de faire remarquer que $A$ peut être considéré ( par $B$ ) comme autorisé à des affirmations auxquelles il n'est pas engagé - il s'agit des conclusions que $B$ peut être autorisé à tirer, mais auxquelles $A$ ne s'est pas encore engagé. Par exemple, $A$ pourrait avoir affirmé que «la température est descendue et le ciel est nuageux». $B$ pourrait ensuite attribuer l'autorisation de $A$ quant à l'engagement envers l'affirmation «il va bientôt pleuvoir» même si $B$ n'attribue pas cet engagement à $A$. Si cet engagement-ci n'est pas déduit à partir des inférences engageantes issues

60 Nous utiliserons «p» à la fois comme un nom pour désigner la phrase que $A$ asserte et comme un nom pour qualifier l'engagement que $A$ reconnaît en énonçant cette assertion. 
d'une affirmation de $A$, mais à partir d'inférences permissives ( $A$ a obtenu, selon celui qui tient le pointage, l'autorisation à asserter cet engagement), alors nous avons de bonnes raisons inductives de croire qu'il va bientôt pleuvoir.

$B$ doit alors, pour finir, évaluer l'autorisation de $A$ quant à l'affirmation que $p$, en examinant les inférences valides dans lesquelles $p$ apparaît comme conclusion et comme prémisse et auxquelles $A$ est engagé et autorisé. $A$ peut s'avérer autorisé à affirmer $p$ de différentes manières. Tout d'abord, $A$ pourrait évaluer la possibilité d'une autorisation par défaut à $p$. Il s'agit du type d'autorisation par défaut caractéristique des comptes rendus d'observation où quiconque qui attribue une telle autorité admet implicitement la fiabilité du rapporteur (dans ces circonstances, et à l'égard de ce contenu). Traiter quelqu'un comme un rapporteur fiable, c'est considérer que l'engagement de ce rapporteur suffit pour que ce rapporteur soit autorisé à cet engagement (Brandom, 2010, p. 368). Ensuite, $A$ pourrait être autorisé à affirmer $p$ dans la mesure où, à titre de conséquence d'autres engagements, $p$ suit en vertu d'inférences engageantes ou d'inférences permissives issues d'autres engagements que $A$ est autorisé à asserter. Enfin, $A$ pourrait être autorisé à asserter $p$ au nom d'un témoignage. Autrement dit, $A$ pourrait reconnaître $p$ dans la mesure où $A$ attribue l'engagement et l'autorisation à asserter $p$ à un autre interlocuteur qui aurait asserté $p$ lors d'une étape précédente et de qui $A$ hérite l'autorisation à $p$. Si $B$ considère que $A$ est autorisé à $p$ par l'un ou l'autre de ces mécanismes d'héritage ou de défaut, alors $B$ considérera que $A$ a avec succès autorisé d'autres personnes à cette affirmation. $B$ considère alors les autres membres de la conversation (y compris lui-même) comme libres de reconnaître le même engagement et, si c'est effectivement le cas pour l'un d'entre eux, celui-ci héritera l'autorisation par voie testimoniale. Cependant, si un autre joueur, disons $C$, considère que $A$ n'est pas autorisé à $p$, alors celui-ci peut soit se retirer du jeu de pointage et attendre la prochaine assertion, soit formuler une assertion incompatible et mettre au défi l'autorité testimoniale de l'assertion de $A$. La portée propre à un tel défi est qu'il met en cause les attributions d'autorisation par défaut là où l'assertion à l'origine du défi est une assertion à laquelle l'auteur du défi est, au moins à première vue, autorisé. Le fait que $B$ considère comme réussi le défi lancé par $C$ à l'assertion de $A$ consiste en ceci que $B$ y répond en suspendant ${ }^{61}$ son attribution d'autorisation à $A$ pour cette affirmation en l'attente d'une

61 Brandom (2010, p. 373-374) introduit cette idée de rétractation afin de corriger la carte de pointage (deontic scoreboard) de chacun des marqueurs. Les désaveux, dit-il, nous permettent de répudier un engagement précédemment contracté, ou de signaler que l'on ne reconnaît pas un tel engagement. Si le désaveu est considéré comme étant réussi et est approuvé par $B$, alors $B$ restitue à $A$ toutes les attributions d'autorisations qu'il lui avait retirées. $A$ reconnaît et répare son erreur afin que la carte de pointage soit réajustée. Mais le désaveu peut aussi 
justification par $A$ de celle-ci. Cela rend cette assertion indisponible (selon la carte de pointage tenue par $B$ ) pour d'autres interlocuteurs qui, autrement, auraient pu hériter d'autorisations à des engagements à l'égard du même contenu.

\section{Conclusion}

En critiquant Brandom du point de vue de l'interaction, Habermas semble réduire la communication à son premier niveau strictement dialogique et tend ainsi à ignorer le second niveau réflexif de la pratique discursive, centrale au JPDR. Brandom concède pourtant bien à Habermas que, dans son modèle communicationnel, la référence au rôle de la seconde personne repose sur l'attitude observationnelle de la troisième personne ou sur celle d'un marqueur qui interprète les performances d'autrui mais n'y voit aucun conflit. En effet, dans ce jeu de marquage au score, tout agent impliqué dans la conversation est un marqueur discursif qui enregistre ses propres statuts déontiques dotés de contenu propositionnel ainsi que ceux des autres. Saisir le contenu sémantique exprimé par l'énonciation affirmative d'une phrase requiert donc l'aptitude à déterminer à la fois ce qui suit d'une assertion (étant donné les autres engagements que le marqueur attribue à l'auteur de l'assertion) et ce qui suit de cette assertion (étant donné les autres engagements que contracte le marqueur), mais implique essentiellement des évaluations à partir des perspectives différentes des interlocuteurs qui marquent au score et qui ont des engagements d'arrière-plan différents. Aussi, un individu ne peut connaître à lui seul toutes les inférences. Le contenu qu'il exprime à partir de son arrière-plan inférentiel est toujours compris et interprété à partir d'un autre arrière-plan inférentiel, lui aussi partiel. Il existe donc toujours un écart entre l'arrière-plan inférentiel d'un locuteur, l'arrière-plan inférentiel du marqueur, et l'arrière-plan général de la communauté. Personne ne connaît et ne possède cet arrière-plan inférentiel général. Il n'apparaît que dans la manière dont nous comprenons et enrichissons, à notre façon, ce que dit autrui sans entraver la communication : nous ne modifions pas le contenu de ce que dit autrui, nous changeons la perspective et l'arrière-plan de ce qu'il dit. La perspective d'autrui est placée dans notre perspective inférentielle et est susceptible d'être interprétée différemment. C'est dans ces changements de perspective que réside l'objectivité de ce que nous disons. La structure sociale sur le mode du

(suite)

échouer, à savoir : $A$ peut acquérir soit directement un engagement à l'égard de $p$, en avouant $p$, soit indirectement, au titre de conséquence d'un engagement à l'égard de $q$, dont $p$ suit en vertu d'une inférence valide et préservant l'engagement (selon $B$ ). Dans ce cas, le désaveu de $p$ par $A$ ne peut réussir (selon $B$ ) que si $A$ est également disposé à désavouer $q$. Mais si $A$ persiste à asserter $q$, alors cet engagement est incompatible avec le désaveu, et le désaveu de $p$ ne restitue aucune autorisation à $A$. $A$ doit donc se rétracter. 
«je-tu» de Brandom ne se limite donc pas à son rôle interactif, mais met en évidence, par la symétrie des perspectives dans ce jeu de pointage, le processus discursif au sein duquel autrui peut aussi bien posséder une perspective inférentielle différente de la nôtre que discerner dans le contenu propre à cette perspective un aspect représentationnel qui nous est commun mais que nous exprimons différemment.

\section{Références bibliographiques}

Bourdieu, Pierre

1990 The Logic of Practice, Cambridge, Polity Press.

Brandom, Robert

1983 «Asserting», Noûs, vol. 17, no 4, p. 637-650.

Brandom, Robert

1997 «Replies», Philosophy and Phenomenological Research, vol. 57, $\mathrm{n}^{\circ} 1$, p. 189-204.

Brandom, Robert

2000a «Facts, Norms, and Normative Facts: A reply to Habermas», European Journal of Philosophy, vol. 8, $\mathrm{n}^{\circ}$ 3, p. 356-374.

Brandom, Robert

2000b «Quelques thèmes pragmatistes dans l'idéalisme de Hegel : Négociation et administration dans la conception hégélienne de la structure et du contenu des normes conceptuelles», Philosophiques, vol. $27, n^{\circ} 2$, p. 213-261.

Brandom, Robert

2002 Tales of the Mighty Dead. Historical Essays in the Metaphysics of Intentionality, Cambridge (MA), Harvard University Press.

Brandom, Robert

2009 L'articulation des raisons : une introduction à l'inférentialisme, Paris, Cerf.

Brandom, Robert

2010 Rendre explicite : raisonnement, représentation et engagement discursif, vol. I [1994], sous la direction d'Isabelle Thomas-Fogiel, Paris, Cerf.

Brandom, Robert

2011 Rendre explicite : raisonnement, représentation et engagement discursif, vol. II [1994], sous la direction d'Isabelle Thomas-Fogiel, Paris, Cerf.

Church, Alonzo

1956 Introduction to Mathematical Logic, Princeton (NJ), Princeton University Press.

Davidson, Donald

2001 Subjective, Intersubjective, Objective, Oxford, Oxford University Press. Dummett, Michael

1978 Truth and Other Enigmas, Londres, Duckworth. 
Dummett, Michael

1981 The Interpretation of Frege's Philosophy, Londres, Duckworth.

Fontaine, Matthieu et Juan Redmont

2008 Logique dialogique : une introduction, vol. I : Méthode de dialogue : règles et exercices, Londres, College Publications.

Gentzen, Gerhard

1935 «Investigations into Logical Deduction», American Philosophical Quaterly, vol. I, no 4, p. 288-306.

Gentzen, Gerhard

1969 The Collected Papers of Gerhard Gentzen, Amsterdam, North Holland. Habermas, Jürgen

1971 «Conférences Christian-Gauss, contribution à la fondation de la sociologie sur une théorie du langage», dans Parcours 1 (1971-1989), Paris, Gallimard, 2018, p. 29-157.

Habermas, Jürgen

1976 «What Is Universal Pragmatics?», dans On the Pragmatics of Communication, Cambridge (MA), MIT Press, 1998a, p. 21-103.

Habermas, Jürgen

1978 Raison et légitimité. Problèmes de légitimation dans le capitalisme avancé, Paris, Payot.

Habermas, Jürgen

1986 «Communicative Rationality and The Theories of Meaning and Action», dans On the Pragmatics of Communication, Cambridge (MA), MIT Press, 1998a, p. 183-213.

Habermas, Jürgen

1987 Logique des sciences sociales, Paris, Presses universitaires de France. Habermas, Jürgen

1988 «Actions, actes de Parole, interactions médiatisées par le langage et le monde vécu», dans Parcours 1 (1971-1989), Paris, Gallimard, 2018, p. 273-325.

Habermas, Jürgen

1992 De l'éthique de la discussion, Paris, Flammarion.

Habermas, Jürgen

1996 Between Facts and Norms, Cambridge (MA), MIT Press.

Habermas, Jürgen

1998a On the Pragmatics of Communication, Cambridge (MA), MIT Press.

Habermas, Jürgen

1998b The Inclusion of the Other, Cambridge (MA), Polity Press.

Habermas, Jürgen

1999 Morale et communication, Paris, Champs-Flammarion.

Habermas, Jürgen

2000 «From Kant to Hegel: On Robert Brandom's Pragmatic Philosophy of Language», European Journal of Philosophy, vol. 8, n ${ }^{\circ}$ 3, p. 322-355. 
Habermas, Jürgen

2001 «De Kant à Hegel : la pragmatique linguistique de Robert Brandom», dans Vérité et justification, chapitre 3, Paris, Gallimard, p. 81-124.

Habermas, Jürgen

2003 Truth and Justification, Cambridge (MA), MIT Press.

Habermas, Jürgen

2005 «À propos de la différenciation architectonique de la discussion. À grande controverse, petite réplique», dans Parcours 2, Tome 2 (19902017), Paris, Gallimard, 2018, p. 117-142.

Habermas, Jürgen

2009 «Cinq introductions à des monographies non écrites, Tome II. Théorie de la rationalité et théorie du langage», dans Parcours 2 (1990-2017), Paris, Gallimard, 2018, p. 196-216.

Habermas, Jürgen

2018 Parcours 1 (1971-1989) et Parcours 2 (1990-2017), trad. de l'allemand par Ch. Bouchindomme, R. Rochlitz, F. Joly et V. Pratt, Paris, Gallimard.

Kant, Emmanuel

1944 Critique de la raison pure, trad. de l'allemand par A. Tremesaygues et B. Pacaud, Paris, Presses universitaires de France.

Lewis, David

1979 «Scorekeeping in a Language Game», Journal of Philosophical Logic, vol. $8, n^{\circ} 1$, p. 339-359.

Lorenz, Kuno

1981 «Dialogical Logic», dans W. Marciszewski, dir., Dictionary of Logic as Applied in the Study of Language, La Haye, Martinus Nijhoff, p. 117125.

Lorenzen, Paul et Kuno Lorenz

1978 Dialogische Logik, Darmstadt, Wissenschaftliche Buchgesellschaft. MacFarlane, John

2010 «Pragmatism and Inferentialism», dans B. Weiss et J. Wanderer, dir., Reading Brandom: On Making It Explicit, Londres, Routledge, p. 81-95. MacFarlane, John

2011 «What is Assertion?» dans J. Brown et H. Cappelen, dir., Assertion, Oxford, Oxford university Press, p. 76-96.

Marion, Mathieu

2012 «Game Semantics and The Manifestation Thesis», dans M. Marion, G. Primiero et S. Rahman, dir., The Realism-Antirealism Debate in the Age of Alternative Logics, Pays-Bas, Springer, p. 141-168.

McDowell, John

1997 «Brandom on Representation and Inference», Philosophy and Phenomenological Research, vol. 57, $\mathrm{n}^{\mathrm{o}}$ 1, p. 157-162.

Rahman, Said et Laurent Keiff

2005 «How to Be a Dialogician», dans D. Vanderveken, dir., Logic, Thought and Action, Dordrecht, Springer, p. 359-408. 
Rorty, Richard

1997 «What Do You Do When They Call You a "Relativist"?» Philosophy and Phenomenological Research, vol. 57, $\mathrm{n}^{\mathrm{o}}$ 1, p. 173-177.

Rorty, Richard

2000a «Response to Habermas», dans Robert Brandom, dir., Rorty and His Critics, Oxford, Blackwell, p. 56-64.

Rorty, Richard

$2000 \mathrm{~b}$ «Response to Brandom», dans Robert Brandom, dir., Rorty and His Critics, Oxford, Blackwell, p. 183-190.

Scharp, Kevin

2003 «Communication and Content: Circumstances and Consequences of the Habermas-Brandom Debate», International Journal of Philosophical Studies, vol. 11, $\mathrm{n}^{\mathrm{o}} 1$, p. 43-61.

Scharp, Kevin

2005 «Scorekeeping in a Defective Language Game», Pragmatics and Cognition, vol. 13, $\mathrm{n}^{\mathrm{o}}$ 1, p. 203-226.

Sellars, Wilfrid

1953 «Inference and Meaning», Mind, vol. 62, n 247, p. 313-338.

Sellars, Wilfrid

1956 Empirisme et philosophie de l'esprit, Combas, L'Éclat.

Strydom, Piet

2006 «Intersubjectivity - Interactionist or Discursive? Reflections on Habermas' Critique of Brandom», Philosophy \& Social Criticism, vol. $32, n^{\circ} 2$, p.155-172.

Swindal, James

2007 «Can a Discursive Pragmatism Guarantee Objectivity? Habermas and Brandom on the Correctness of Norms», Philosophy \& Social Criticism, vol. 33, $\mathrm{n}^{\mathrm{o}}$ 1, p. 113-126.

Trafford, John

2017 «Dialogue and Refutation», dans L. Magnani, dir., Meaning in Dialogue: An Interactive Approach to Logic and Reasoning, Londres, Springer («Studies in Applied Philosophy, Epistemology and Rational Ethics»), p. 79-108.

Wittgenstein, Ludwig

2001 Tractatus Logico-Philosophicus, trad. de l'allemand par Gilles-Gaston Granger, Paris, Gallimard.

Wittgenstein, Ludwig

2005 Recherches philosophiques, Paris, Gallimard. 